\title{
Best practices on immunomodulators and biologic agents for ulcerative colitis and Crohn's disease in Asia
}

Choon Jin Ooi ${ }^{1,2 *}$, Ida Hilmi ${ }^{3 *}$, Rupa Banerjee ${ }^{4}$, Sai Wei Chuah ${ }^{5}$, Siew Chien $\mathrm{Ng}^{6}$, Shu Chen Wei ${ }^{7}$, Govind K Makharia ${ }^{8}$, Pises Pisespongsa ${ }^{9}$, Min Hu Chen ${ }^{10}$, Zhi Hua Ran ${ }^{11}$, Byong Duk Ye ${ }^{12}$, Dong Il Park ${ }^{13}$, Khoon Lin Ling ${ }^{2}$, David Ong ${ }^{14}$, Vineet Ahuja ${ }^{7}$, Khean Lee $\mathrm{Goh}^{15}$, Jose Sollano ${ }^{16}$, Wee Chian Lim $^{17}$, Wai Keung Leung ${ }^{18}$, Raja Affendi Raja Ali ${ }^{19}$, Deng Chyang Wu ${ }^{20}$, Evan Ong ${ }^{16}$, Nazri Mustaffa ${ }^{21}$, Julajak Limsrivilai ${ }^{22}$, Tadakazu Hisamatsu ${ }^{23}$, Suk Kyun Yang ${ }^{24}$, Qin Ouyang ${ }^{25}$, Richard Geary ${ }^{26}$, Janaka H De Silva ${ }^{27}$, Rungsun Rerknimitr ${ }^{28}$, Marcellus Simadibrata ${ }^{29}$, Murdani Abdullah ${ }^{30}$, Rupert WL Leong ${ }^{31}$, on behalf of the Asia Pacific Association of Gastroenterology (APAGE) Working Group on Inflammatory Bowel Disease and Asian Organization for Crohn's and Colitis

${ }^{1}$ Department of Gastroenterology and Hepatology, Singapore General Hospital, Singapore; ${ }^{2}$ Duke-NUS Medical School, Singapore; ${ }^{3}$ Department of Medicine, Faculty of Medicine, University Malaya Medical Centre, Kuala Lumpur, Malaysia; ${ }^{4}$ Asian Institute of Gastroenterology, New Delhi, India; ${ }^{5}$ Gleneagles Medical Centre, Singapore; ${ }^{6}$ Department of Medicine and Therapeutics, The Chinese University of Hong Kong, Hong Kong; ${ }^{7}$ Department of Internal Medicine, National Taiwan University Hospital, Taipei, Taiwan; ${ }^{8}$ Department of Gastroenterology and Human Nutrition, All India Institute of Medical Sciences, New Delhi, India; ${ }^{9}$ Gastroenterology and Hepatology, Bumrungrad International University, Bangkok, Thailand; ${ }^{10}$ Division of Gastroenterology, The First University Hospital, Sun Yat-sen University, Guangzhou; ${ }^{11}$ Department of Gastroenterology and Hepatology, Renji Hospital, School of Medicine, Shanghai Jiao Tong University, Shanghai, China; ${ }^{2}$ Department of Gastroenterology and IBD Center, University of Ulsan College of Medicine, Seoul; ${ }^{13}$ Department of Internal Medicine, Kangbuk Samsung Hospital, Sungkyunkwan University School of Medicine, Seoul, Korea; ${ }^{14}$ Division of Gastroenterology and Hepatology, National University Hospital of Singapore, University Medicine Cluster, Singapore, ${ }^{15}$ University of Malaya Specialist Centre, Kuala Lumpur, Malaysia; ${ }^{16}$ Department of Medicine, University of Santo Tomas, Manila, Philippines; ${ }^{17}$ Department of Gastroenterology and Hepatology, Tan Tock Seng Hospital, Singapore; ${ }^{18}$ Division of Gastroenterology and Hepatology, Department of Medicine, University of Hong Kong, Hong Kong; ${ }^{19}$ Faculty of Medicine, UKM Medical and Specialist Centres, The National University of Malaysia, Kuala Lumpur, Malaysia; ${ }^{20}$ Department of Medicine, Kaohsiung Medical University, Kaohsiung, Taiwan; ${ }^{21}$ Department of Internal Medicine, School of Medical Sciences, Health Campus, Sains University, Kubang Kerian, Malaysia; ${ }^{22}$ Department of Medicine, Faculty of Medicine, Siriraj Hospital, Mahidol University, Bangkok, Thailand; ${ }^{23}$ The Third Department of Internal Medicine, Kyorin University School of Medicine, Mitaka, Japan; ${ }^{24}$ Department of Gastroenterology, University of Ulsan College of Medicine, Seoul, Korea; ${ }^{25}$ Department of Gastroenterology, West China Hospital, Sichuan University, Chengdu, China; ${ }^{26}$ Department of Medicine, University of Otago, Christchurch, New Zealand, ${ }^{27}$ Faculty of Medicine, University of Kelaniya, Dalugama, Sri Lanka; ${ }^{28}$ Department of Medicine, Chulalongkorn University, Bangkok, Thailand; ${ }^{29}$ Department of Internal Medicine, Faculty of Medicine, University of Indonesia, Jakarta; ${ }^{30}$ Division of Gastroenterology, Department of Internal Medicine, Cipto Mangunkusumo National Hospital, Jakarta, Indonesia; ${ }^{31}$ Gastroenterology and Liver Services, Concord Hospital, Sydney, Australia

Received March 20, 2019. Revised April 10, 2019. Accepted April 15, 2019. Correspondence to Choon Jin Ooi, Department of Gastroenterology and Hepatology, Singapore General Hospital, 6 Napier Road, \# 10-02, Gleneagles Medical Centre, Singapore 258499, Singapore. Tel: +65-6471-2500, Fax: +65-6471-2578, E-mail: eddyooi@duke-nus.edu.sg

ORCID Choon Jin Ooi (https://orcid.org/0000-0001-8961-5455), Ida Hilmi (https://orcid.org/0000-0001-7091-0032)

*These authors contributed equally to this study.

The consensus was developed by Asia Pacific Association of Gastroenterology Working Group on Inflammatory Bowel Disease and Asian Organization for Crohn's and Colitis, and are being published simultaneously in the Intestinal Research and Journal of Gastroenterology and Hepatology.
The Asia-Pacific Working Group on inflammatory bowel disease (IBD) was established in Cebu, Philippines, under the auspices of the Asian Pacific Association of Gastroenterology with the goal of improving IBD care in Asia. This consensus is carried out in collaboration with Asian Organization for Crohn's and Colitis. With biologic agents and biosimilars becoming more established, it is necessary to conduct a review on existing literature and establish a consensus on when and how to introduce biologic agents and biosimilars in the conjunction with conventional treatments for ulcerative colitis (UC) and Crohn's disease (CD) in Asia. These statements also address how pharmacogenetics influence the 
treatments of UC and CD and provide guidance on response monitoring and strategies to restore loss of response. Finally, the review includes statements on how to manage treatment alongside possible hepatitis B and tuberculosis infections, both common in Asia. These statements have been prepared and voted upon by members of IBD workgroup employing the modified Delphi process. These statements do not intend to be all-encompassing and future revisions are likely as new data continue to emerge. (Intest Res 2019;17:285-310)

Key Words: Inflammatory bowel disease; Crohn disease; Colitis, ulcerative; Infliximab; Adalimumab

\section{INTRODUCTION}

The prevalence of IBD in Asia, whilst not having achieved the same prevalence as in countries of the western hemisphere, is on the rise. The Asia-Pacific Working Group on IBD previously published 2 review papers that addressed the definition, diagnosis, epidemiology ${ }^{1,2}$ as well as the management of IBD. Corticosteroids and mesalazine remain important agents used in the induction of remission in IBD throughout Asia. Given the endemicity of hepatitis and tuberculosis (TB) in Asia, the use of immunomodulators and biologic agents requires considerations not taken into account in the rest of the world.

Immunomodulators, which include methotrexate and the thiopurines, modify the immune system with the aim of inducing and maintaining remission. Their effects are systemic and not as targeted as the biologic agents. The thiopurines are generally slow acting but there is greater experience in their use. Biologic agents are a class of drugs produced by living organisms such as bacteria or mammalian cells in culture. These drugs consist of large and complex molecules and are often protein-based. They are relatively new in many parts of Asia, and hence, more guidance is required in their use as monotherapy agents or in combination therapy with immunomodulators. Biologic agents also provide a more targeted approach to reducing inflammation in the form of monoclonal antibodies against TNF- $\alpha$ (anti-TNF- $\alpha$ ), interleukins (ILs) and integrins. However, due to the high cost of these drugs, there are constraints in making them widely accessible in Asia.

This paper will review how immunomodulators can be coupled with biologic agents as a treatment protocol for IBD. The paper will cover indications for initiation of such therapy, disease monitoring, individualized dosing of these drugs and exit strategies. Pharmacogenetics or precision medicine have helped optimized the drug armamentarium for IBD. For patients being considered for thiopurines, thiopurine methyltransferase (TPMT) polymorphisms, while important for Western populations, may not be as relevant in Asia. However, nucleoside diphosphate linked moiety X-type motif 15 (NUDT15) genotyping is encouraged, where available, prior to initiation of azathioprine. Further to the Asian context of this review, endemic diseases such as $\mathrm{TB}$ and hepatitis $\mathrm{B}$ require special attention prior to treatment. Rigorous screening protocols and the use of prophylaxis measures, where appropriate, will be addressed.

\section{METHODS}

A modified Delphi process ${ }^{3}$ was adopted to develop the consensus statements according to their clinical importance within the Asia-Pacific region. A steering committee (C.J.O., I.H., R.B., R.W.L., S.W.C., S.C.N., P.P., and V.A.) generated a list of statements and circulated it electronically to Consensus Group members. These statements were presented to the Consensus Group panel for discussion, revision, and voting. A passwordsecured website was populated with the relevant literature assembled by the steering committee. A systematic literature review was conducted to identify and grade the available evidence to support each statement. The literature search was conducted in the English language publications indexed in the MEDLINE, Embase, and the Cochrane Trials Registry databases, and limited to those in human subjects. Regional and international consensus statements and guidelines on IBD were examined. Relevant literature from the Asia-Pacific region was of particular interest.

The categorization of evidence, classification of recommendation, and voting schema were according to the Canadian Task Force on the Periodic Health Examination (Table 1). ${ }^{4}$ Consensus was achieved when $80 \%$ or more of votes were either accepted "completely" or "with some reservation." A statement was refuted when $80 \%$ or more of voting members rejected a statement "completely" or "with some reservation." Every statement was then graded to indicate the level of evidence available and the strength of recommendation. 
Table 1. Quality of Evidence, Classification of Recommendation, and Voting on Recommendations

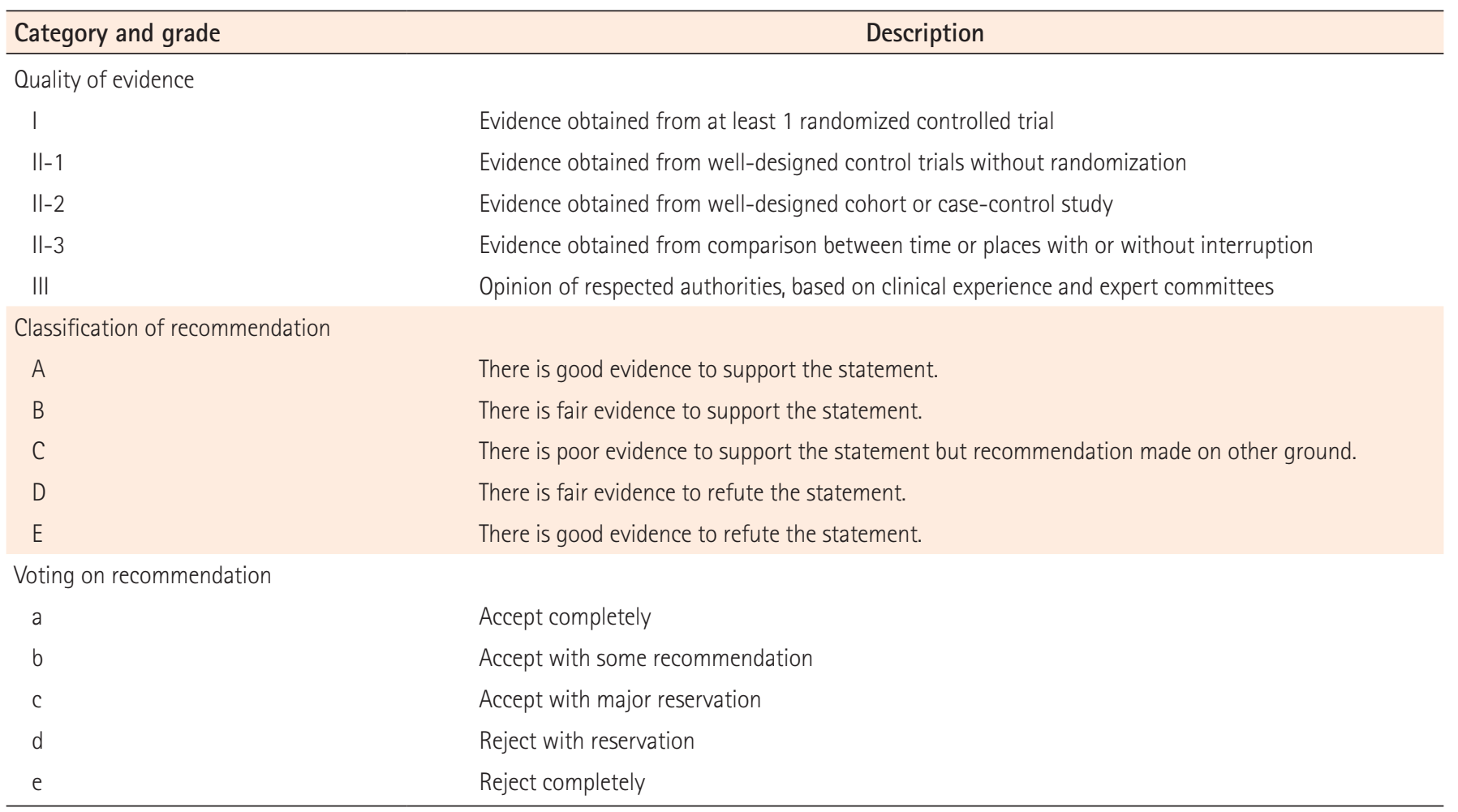

\section{Membership of the Consensus Group}

Voting members of the Consensus Group were selected using the following criteria: (1) Demonstration of knowledge and expertise in IBD through publication/research or participation in national or regional guideline development; (2) Geographical representation of the Asia-Pacific countries; (3) Diversity of views and expertise in healthcare system (including colorectal surgeon, pathologist, pharmacist, nurse practitioners). Voting was limited, however, to clinicians.

Representative countries included Malaysia, Thailand, Sri Lanka, India, China, Hong Kong, Taiwan, Philippines, Indonesia, Australia, New Zealand, Japan, South Korea, and Singapore.

\section{Voting, Delphi Process, and General Organization of the Consensus}

Voting was conducted anonymously at all times. The first vote was conducted by the entire Consensus Group electronically by email. Relevant literature was then made available on a secured website for review by all voters, and a second round of voting was undertaken, during which members could modify their first-round selections, if required. A third and final vote was held thereafter during a face-to-face meeting. Statements that could not reach consensus were discussed and either modified or rejected. Each statement was graded to indicate the level of evidence available and the strength of recommendation by using the Canadian Task Force Guidelines on the Periodic Health Examination. ${ }^{4}$ Improvement to the wording of the statements was permitted following an open discussion. The full Consensus Group meeting was held in August 2017 in Penang, Malaysia. Representatives attended from Asia-Pacific countries that included Australia, Hong Kong, India, China, South Korea, Malaysia, Philippines, Singapore, Taiwan, and Thailand.

\section{RESULTS}

\section{Part A: Biologic Therapy-Who to Consider for Biologic Agents and When to Start?}

\section{Statement 1}

Biologic therapy (anti-TNFs, anti-integrins, anti-IL12/23) should be initiated for the treatment of moderate-to-severe CD. This includes corticosteroid or immunosuppressant refractory/intolerant disease and corticosteroid dependence. - Level of agreement: (a) 100\%, (b) 0\%, (c) 0\%, (d) 0\%, (e) $0 \%$. - Quality of evidence: I

- Classification of recommendation: A 
Biologic therapy can be initiated in moderate to severe inflammatory active disease if conventional therapy fails or if it is not tolerated. ${ }^{5}$ The conventional therapy for moderate to severe luminal CD include mesalazines and corticosteroids as the first line therapy. Immunomodulators are commenced as corticosteroid-sparing agents in case of recurrent relapses, corticosteroid dependence or corticosteroid refractory states. Several studies have provided evidence that the use of biologic agents with or without immunomodulators in moderate to severe $\mathrm{CD}$ can reduce inflammation and flares. The use of biologic agents promotes mucosal healing, as well as lower the rates of hospitalization and surgery. ${ }^{6-8}$ In an European multicenter trial, histological remission and mucosal healing were demonstrated with infliximab in $\mathrm{CD} .{ }^{9}$ Lichtenstein et al. ${ }^{7}$ reported that in patients enrolled in the ACCENT II trial, infliximab reduced hospitalizations, surgeries, and procedures. The EXTEND trial showed that adalimumab effectively induced and maintained mucosal healing. ${ }^{10}$

Anti-TNFs have been extensively evaluated for the induction of remission and maintenance in CD in several randomized control trials and meta-analyses. Infliximab was the first biologic agent and has the maximum published data with regards to the use of biologic agents in CD. The ACCENT 1 trial demonstrated the efficacy of infliximab as a maintenance therapy in patients who responded to an initial dose of infliximab and discontinued corticosteroids whilst maintaining prolonged remission. ${ }^{11}$ In the CHARM trial, adalimumab was found to be effective in maintaining remission in moderate to severe CD through to 56 weeks. ${ }^{12}$ The Precise 1 and Precise 2 trials demonstrated that certolizumab pegol was able to induce and maintained remission in moderate to severe CD. ${ }^{13,14}$ However, it needs to be mentioned that the patient cohorts in these studies have been heterogeneous, including patients with both corticosteroid naïve and corticosteroid dependent or resistant disease, and the latter studies included patients that had failed prior anti-TNF therapy. ${ }^{15}$

The anti-integrin antibody, vedolizumab has shown efficacy in the management of moderate to severe CD in both anti-TNF naïve subjects and those that had failed anti-TNF treatment in the GEMINI II ${ }^{16}$ and GEMINI III studies. ${ }^{17}$ Natalizumab, an earlier anti-integrin antibody, is not widely used due to the increased risk of progressive multifocal leukoencephalopathy. ${ }^{17,18}$ Ustekinumab is a monoclonal antibody directed against the p40 subunit of IL-12 and IL-23. It effectively induces and maintains remission in moderate to severe luminal CD including those who had failed anti-TNFs in the pivotal UNITI I/II and
IM-UNITI clinical trials. ${ }^{19}$ Currently there is no head-to-head data comparing the currently available biologic therapies. The choice of biologic therapy, therefore, should take into consideration patient and disease characteristics, reimbursement policies/cost, risk for adverse effects, presence of extraintestinal manifestations, possibility of pregnancy, as well as patient's preference in terms of the route of administration.

\section{Statement 2}

Although early use of biologic therapy has been shown to improve clinical outcome, the top down approach cannot be recommended in all patients with active CD. An accelerated step up to biologic therapy can be suggested after consideration of high risk factors and predictors of poor outcome.

- Level of agreement: (a) 66.67\%, (b) 33.33\%, (c) 0\%, (d) 0\%, (e) $0 \%$.

- Quality of evidence: I

- Classification of recommendation: A

The traditional therapeutic approach is a step-up treatment strategy which consists of initiating biologic therapy only after conventional therapy has failed to control the disease. However, this approach has raised concerns that delaying biologic therapy may result in irreversible intestinal strictures and fistulizing disease. Accordingly, a top-down treatment strategy with the early use of biologic agents was suggested. ${ }^{20,21}$ The top-down approach has been shown to be more effective in maintaining remission, decreasing the rate of relapse, reducing the administration of corticosteroids, preventing the occurrence of complications and minimizing surgeries in the long term as well as improving the quality of life. ${ }^{20}$ However, there are other factors that need to be considered for the topdown approach, particularly within Asia.

In many Asian countries, where there is a high prevalence of latent TB, the use of anti-TNFs with top-down approach may be risky. Navarra et al. ${ }^{22}$ assessed the risk of TB in patients treated with anti-TNF agents in Asia and reported a substantially higher number of patients at risk compared to Western Europe and North America. In addition to TB, other opportunistic infections and malignancy remain a major concern especially when biologic agents are introduced early and for a prolonged duration. Additionally, top-down treatment may not be cost-effective if this approach is considered in every patient with $\mathrm{CD}$, especially in countries where most of the people are uninsured. ${ }^{23}$ The proportion of CD patients 
under treatment with biologic agents in Asian countries differ markedly and is as high as $30 \%$ to $40 \%$ in Japan, where the government pays for all the expenses for IBD, down to $1 \%$ in India, where patients have to pay for biologic agents themselves. ${ }^{23}$ Primary top-down therapy also risks over-treatment. Chen et al. ${ }^{20}$ and Lin et al. ${ }^{24}$ reported that as high as $30 \%$ of patients might have been over-treated with this approach. Identification of subgroups that would benefit the top-down therapeutic approach that maximizes the treatment benefitrisk profile is required..$^{15,24}$

The alternative accelerated step-up approach is one where biologic agents are introduced early in patients with high risk factors for failure with conventional treatments to permit better clinical outcomes. ${ }^{25}$ Various studies have been published to identify predictors for poor outcomes in CD so as to categorize patients in terms of risk. Beaugerie et al. ${ }^{26}$ reported that for patients below the age of 40 years, the presence of perianal disease, and the initial requirement of corticosteroids are factors predictive of subsequent 5 -year disabling course. Sands et al. ${ }^{27}$ reported that the use of corticosteroids in the first 6 months of diagnosis was associated with 4 -fold increased risk of surgery. Other clinical markers include stricturing or penetrating disease behavior, extensive disease, small bowel disease and perianal disease. ${ }^{28,29}$ Smoking was associated with poorer outcomes such as the development of strictures, fistulae, increased risk for surgery and the need for corticosteroids and/or immunomodulators. ${ }^{28,30}$ The presence of risk factors would place the patient in the moderate/high-risk category, whereas limited anatomic involvement with superficial ulcers, the absence of stricturing/penetration behavior, perianal disease/rectal disease and prior surgical resection would place the patient in a low-risk category. ${ }^{31}$

Risk stratification helps in identifying patients at risk of poor prognosis and guides clinicians in identifying patients who might benefit from early aggressive therapy while at the same time avoiding over treatment in those with mild disease. An Asian study was undertaken by $\mathrm{Oh}$ et al. ${ }^{32}$ which reported that Korean patients with poor prognostic factors treated with antiTNFs or immunomodulators within 2 years of diagnosis is associated with better clinical outcomes than later treatment. The poor prognostic factors include patients younger than 40 years of age at diagnosis, treated with systemic corticosteroids within 3 months of diagnosis and had a perianal fistula at the time of diagnosis.

\section{Statement 3}

Combination therapy should be considered in patients naïve to biologic agents, particularly infliximab, at least for the first 6 months to 1 year of therapy but this must be carefully weighed against the risks of infection and malignancy.

. Level of agreement: (a) 45.45\%, (b) 45.45\%, (c) 9.09\%,

(d) $0 \%$, (e) $0 \%$.

- Quality of evidence: II-3

- Classification of recommendation: A

Studies have suggested that early combination therapy of infliximab with azathioprine is more efficacious than monotherapy with infliximab in terms of induction of remission and reduction of corticosteroid use. ${ }^{33,34}$ The SONIC trial demonstrated the superiority of infliximab combination therapy over monotherapy in CD patients naïve both to thiopurines and biologic agents. ${ }^{34}$ Similarly, the UC success study, has also shown that infliximab and azathioprine combination therapy is superior to infliximab or azathioprine alone ${ }^{35}$ However, a metaanalysis reported that combination therapy of anti TNF therapy with immunomodulators is not as beneficial compared to monotherapy in inducing or maintaining a clinical response when anti-TNF therapy is added to an existing immunosuppressive regimen. ${ }^{36}$ Studies have also failed to show the benefit of combination therapy with adalimumab/immunomodulator as compared to adalimumab monotherapy. ${ }^{37}$

The benefits of combination therapy are reduced immunogenicity, increased serum levels and better efficacy of anti-TNF agents. In addition to reducing immunogenicity, immunosuppressants can reverse antidrug antibody formation mostly within 12 months. ${ }^{38,39}$

This must be carefully weighed against the risk of infection, especially in Asian countries where the prevalence of TB is high (see Statements 27-29). Another rare risk is hepatosplenic T-cell lymphoma which has been associated especially with thiopurines. ${ }^{36}$ Currently, there is no data available on combination therapies involving non anti-TNF medications. Evidence on the combination therapy of methotrexate with infliximab is limited, however, available data suggest that combination therapy is no more effective than monotherapy with infliximab. ${ }^{40}$

In a study on immunosuppression withdrawal in CD, continued treatment with immunosuppressives beyond 6 months of combination treatment offered no additional benefit over infliximab monotherapy in patients with CD in stable remission. In a randomized control trial, $1-1.25 \mathrm{mg} / \mathrm{kg} /$ day azathio- 
prine was as effective as full dose azathioprine in terms of preventing clinical relapse after 1 year in IBD patients on remission on combination therapy. Thus, a low dose immunosuppressant for short duration (6-12 months) is an effective strategy for combination therapy. ${ }^{41-43}$

\section{Statement 4 \\ Episodic treatment should be avoided to prevent sensitiza- tion. However, de-escalation or discontinuation of biologic therapy, may be considered in carefully selected cases. \\ - Level of agreement: (a) 90\%, (b) 10\%, (c) 0\%, (d) 0\%, (e) $0 \%$. - Quality of evidence: II \\ . Classification of recommendation: B}

Biologic agents are expensive and thus biologic therapy should not be initiated unless one can afford the treatment for at least 6 to 12 months. For patients who are immunosuppressant resistant or intolerant, treatment, once started, should be prolonged. Single dose or episodic treatment should be strictly avoided as they may lead to antibody-formation resulting in loss of efficacy, increased risk of infusion and possible delayed hypersensitivity reactions. The development of antibodies has been shown to be as high as $30 \%$ to $61 \%$ in patients receiving episodic infliximab compared to $7 \%$ to $10 \%$ in patients with scheduled infliximab infusions. ${ }^{15,44}$ The incidence of antidrug antibodies was lower in patients with concomitant immunomodulators than in those patients without immunomodulators. ${ }^{11,45}$ Sands et al. ${ }^{45}$ reported that the likelihood of infusion reactions was 2 to 3 times higher in patients who were positive for antibodies to infliximab than in those who were negative for antibodies or had inconclusive results. ACCENT 1 trial documented the occurrence of infusion reactions in $16 \%$ of patients positive for antibodies to infliximab compared to $8 \%$ in those without antibodies. ${ }^{11}$

After discontinuation of anti-TNF therapy, relapse rates of $40 \%$ and $50 \%$ over a 2 -year period have been reported by various studies. In many parts of Asia, it is not possible to continue biologic therapy indefinitely due to the high cost of therapy or difficult access to infusion centers. Therefore, discontinuation of biologic therapy may need to be considered if the patient meets certain criteria.

Factors that that may tip a clinician to consider de-escalation of therapy include older patients, limited disease involvement, little or no treatment delay after early diagnosis and good treatment response to stable therapy. De-escalation can also be considered in patients with mucosal healing who are in prolonged remission. A history of cancer or serious infections during biologic therapy may preclude the continuation of such therapy. Young patients, the presence of ileal/peri-anal/extensive disease, previous immunomodulator failure/surgery/antiTNF use and relapsing course are factors that favor the continuation of therapy. ${ }^{46}$ Stopping biologics in patients in deep remission (clinical and endoscopic) is associated with a low chance of relapse (STORI trial). ${ }^{47}$ Dose reduction, lengthening interval of therapy and drug recycling can be a cost-effective strategy in patients in whom stopping treatment is not feasible. ${ }^{48-50}$

\section{Statement 5}

Biologic agents should be used as first-line treatment for complex perianal fistulas in combination with surgical intervention. For simple perianal fistulas, biologic agents are recommended if surgical intervention, antibiotics and immunomodulators fail.

- Level of agreement: (a) 85\%, (b) 15\%, (c) 0\%, (d) 0\%, (e) $0 \%$. - Quality of evidence: I

- Classification of recommendation: A

Perianal fistulas is a significant complication observed in $21 \%$ to $54 \%$ of CD. There is strong data for the use of biologic therapy for perianal disease. Antibiotics with or without local drainage (e.g., using setons) is usually adequate for simple perianal fistulas without active rectal inflammation. For complex CD-related fistulas, there is limited benefit with thiopurines; corticosteroids are ineffective and may worsen the sepsis.

Biologic agents have resulted in a paradigm shift in the management of this CD-related perianal fistulas. The ACCENT II study showed that infliximab at a dose of $5 \mathrm{mg} / \mathrm{kg}$ at 0,2 , and 6 weeks followed by 8 weekly maintenance for 54 weeks resulted a fistula closure of $36 \%$ versus $19 \%$ in the placebo group. ${ }^{45}$ A recent meta-analysis has confirmed the efficacy of all antiTNFs (infliximab, adalimumab and certolizumab pegol). ${ }^{51}$ Subgroup analysis from the GEMINI II study also showed the benefit of vedolizumab in CD-related perianal fistulas. ${ }^{52}$ Currently, there are no head-to-head data comparing the efficacy of the different biologic agents. A small retrospective study did not show a significant difference in recurrence between infliximab and adalimumab. ${ }^{53}$ The management of perianal fistulas requires a multidisciplinary approach and emerging therapies such as intralesional mesenchymal stem cells will further enhance the benefit of biologic agents and reduce the rates of proctectomy and permanent stoma. ${ }^{54}$ 


\section{Statement 6}

Biologic therapy is recommended for the treatment of moderate-to-severe UC if conventional therapy fails.

- Level of agreement: (a) 95\%, (b) 5\%, (c) 0\%, (d) 0\%, (e) $0 \%$.

- Quality of evidence: I

- Classification of recommendation: A

The ACT 1 and 2 studies showed the benefit of infliximab for both induction and maintenance of moderate to severe UC. ${ }^{55}$ Adalimumab has also been found to be efficacious in UC (ULTRA studies), ${ }^{56}$ as has golimumab (PURSUIT studies). ${ }^{57,58}$ The GEMINI I study found vedolizumab to be efficacious in the induction and maintenance of remission in UC. ${ }^{59}$ Biologic agents, therefore, should be initiated in patients not responding to conventional therapy; which is usually defined as disease that is refractory or intolerant to adequate doses of immunosuppressive therapies such as a thiopurine and methotrexate, corticosteroid dependence (recurrence of symptoms on corticosteroid tapering) or corticosteroid refractory disease.

\section{Statement 7}

Infliximab or cyclosporine A should be considered as rescue therapy in patients with acute severe UC that is nonresponsive to intravenous corticosteroids within 3-7 days. - Level of agreement: (a) 86\%, (b) 14\%, (c) 0\%, (d) 0\%, (e) $0 \%$. - Quality of evidence: 1

- Classification of recommendation: A

A randomized study of corticosteroid-refractory severe UC patients evaluated the effect of infliximab rescue therapy on colectomy avoidance. At 3 months, those randomized to infliximab showed a significantly lower colectomy rate than the placebo group ( $29 \%$ vs. $67 \%$ ) with fewer postoperative complications. ${ }^{60}$ Cyclosporine has long been shown as an effective rescue therapy in corticosteroid refractory acute severe colitis. ${ }^{61}$ An open labelled study comparing the 2 drugs did not show any significant difference in outcome ${ }^{62}$ and this was confirmed in the CONSTRUCT study. ${ }^{63}$ Therefore, the choice of treatment is based on factors such as local availability, cost of therapy and physician preference, whether patients have been unsuccessfully treated with thiopurines previously. ${ }^{64}$ Currently there are no data for other biologic agents such as adalimumab, golimumab and vedolizumab and the general consensus is that these drugs do not act rapidly enough to be effective in this setting. Surgery remains a reasonable and cost-effective option, particularly in many parts of Asia.

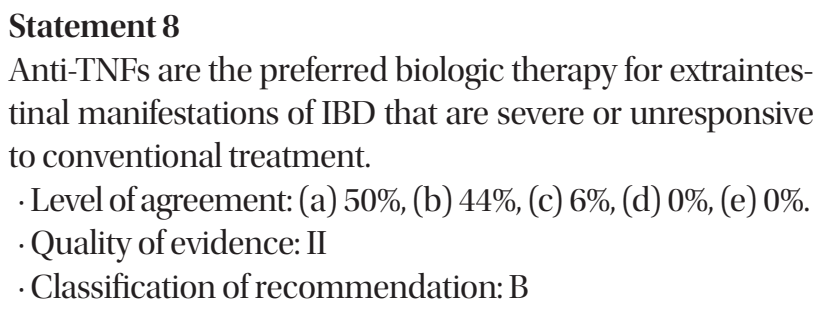

Anti-TNFs are approved for many autoimmune conditions such as rheumatoid arthritis and psoriatic arthropathy and their efficacy in extraintestinal manifestations for IBD, in particular, is well-established. A recently published systemic review has confirmed that anti-TNFs are effective for many of the extra intestinal manifestations including arthropathies, skin manifestations especially pyoderma gangrenosum, ocular manifestations and anaemia. ${ }^{65}$ At present, there is minimal data for ustekinumab and vedolizumab. Although a recent study shown that vedolizumab was effective in reducing extra intestinal manifestations associated with intestinal activity, ${ }^{66}$ more data is required.

\section{Statement 9}

Anti-integrins and anti-IL-12/23 may be associated with a lower risk of $\mathrm{TB}$.

- Level of agreement: (a) 63\%, (b) 37\%, (c) 0\%, (d) 0\%, (e) $0 \%$.

- Quality of evidence: II-2

- Classification of recommendation: B

Anti-TNFs are indicated for those who fail conventional therapy but $\mathrm{TB}$ reactivation remains a concern in TB endemic countries. Prior to starting an anti-TNF, rigorous testing for the exclusion of TB or latent TB is required.

Vedolizumab, on the other hand, is unlikely to reactivate TB. A review by Colombel et al. ${ }^{67}$ of 2,830 patients across 6 IBD clinical trials showed only 4 cases of TB. The real world GETAID study did not show any cases of TB in $173 \mathrm{CD}$ and 121 UC cases in 54 week follow-up. ${ }^{68}$ Similarly, 94 CD and 42 UC patients from the United States treated for either UC or CD did not show any $\mathrm{TB}$ reactivation after 1 year. ${ }^{69}$

Ustekinumab also have decreased sepsis risk and lower potential for TB reactivation than anti-TNFs. In the Psoriasis Longitudinal Assessment and Registry PSOLAR study involving 12,093 patients and 40,388 patient years (PY), overall incidence rates were $0.68 / 100 \mathrm{PY}$ for malignancy, $1.60 / 100 \mathrm{PY}$ for seri- 
ous infection, and 0.46/100 PY for mortality. Unadjusted rates of serious infection for infliximab (2.91/100 PY) and other biologic agents (1.91/100 PY) were numerically higher compared with ustekinumab $(0.93 / 100 \mathrm{PY}) .^{70}$ Tsai et al., ${ }^{71}$ in a review of 3,172 plaque psoriasis patients across 5 phase III trial of ustekinumab, showed no reactivation of LTBI reactivation was observed in patients receiving continuous isoniazid prophylaxis for LTBI. Papp et al. ${ }^{72}$ in an analysis of 2014 PSOLAR data did not identify any serious infection with ustekinumab. In the psoriatic arthritis studies PSUMMIT I and PSUMMIT II, McInnes et al. ${ }^{73}$ and Ritchlin et al. ${ }^{74}$ reported on 615 and 312 subjects respectively. No cases of TB were reported in the followup period of 52 weeks in PSUMMIT I and 60 weeks in PSUMMIT II. There are isolated case reports documenting TB in patients treated with ustekinumab. These involved a case of peritoneal TB and peripheral lymph node reactivation of TB in patients with psoriasis treated with ustekinumab. ${ }^{75,76}$ The relative safety of ustekinumab was again noted when assessed in 1,407 adult patients with moderate to severely active CD in 3 randomized, double-blind, placebo-controlled, parallel-group, multicenter, phase 3 studies (2 induction trials, UNITI-1, UNITI-2; 1 maintenance trial, IMUNITI).

\section{Part B: Biosimilars}

\section{Statement 10 \\ Currently approved biosimilars are as safe and effective as reference products and can be used as induction and main- tenance therapy for both $\mathrm{CD}$ and UC. \\ - Level of agreement: (a) 50\%, (b) 44\%, (c) 6\%, (d) 0\%, (e) $0 \%$. - Quality of evidence: II-2 \\ - Classification of recommendation: B}

A biosimilar medicinal product is an almost an identical copy of an original licensed "reference" biologic agent. Biosimilars in IBD may reduce drug acquisition cost and increase cost-effectiveness and increase capacity for their use. Given the complexity of the structure of monoclonal antibodies, minor structural differences of the drug are unavoidable as the manufacturing process cannot be absolutely controlled. These minor structural changes may theoretically result in changes to the drug's immunogenicity, which may then change its efficacy and safety particularly following one- or multiple switches from the originator produced or from different biosimilars. ${ }^{77,78}$ As such, biosimilars are not generic drugs because they are not identical to the originator biologic agent.
Cohen et al. ${ }^{79}$ conducted a systematic review of switching reference medicines to biosimilars. Ninety studies were identified involving 7 agents that treated 14 disease indications, and enrolled a total of 14,225 individuals. Most of these studies showed that there were no differences in terms of safety, efficacy or immunogenicity after switching patients to biosimilars. These data addressed concerns over immunogenicity, efficacy and safety when switching from an innovator to a biosimilar biologic agent. Prospective and retrospective IBD-specific data showed that switching to biosimilars (mostly CT-P13, also known as Inflectra, Remsima and infliximab-dyyb, the first biosimilar monoclonal antibody) made no significant differences in efficacy, safety and immunogenicity. ${ }^{79}$ The PROSIT-BIO cohort: a prospective observational study of patients with IBD treated with CT-P13 included 313 CD and 234 UC patients. Of these, 97 patients who were on infliximab were switched directly to CT-P13 and comparable outcomes were observed ${ }^{80}$ Schmitz et al. ${ }^{81}$ studied a cohort of 133 IBD patients (64\% CD and 36\% UC) on the infliximab innovator, Remicade which was switched to infliximab biosimilar, Inflectra. No differences in drug levels and disease activity between infliximab innovator and biosimilar were found, indicating that these biosimilars were safe and effective. A post-marketing study from Korea included 176 patients with active moderate-to-severe $\mathrm{CD}$, fistulizing $\mathrm{CD}$, or moderate-to-severe UC treated with biosimilar infliximab (CT-P13) and followed for 30 weeks and found that CT-P13 was well tolerated and efficacious in patients with IBD. ${ }^{82}$

The Food and Drug Administration (FDA) has already approved 4 biosimilars: infliximab-dyyb (Inflectra; Celltrion, Incheon, Korea) and infliximab-abda (Renflexis; Merck KGaA, Darmstadt, Germany), which are biosimilars to infliximab, as well as adalimumab-atto (Amjevita; Amgen Inc., Newbury, CA, USA) and adalimumab-adbm (Cyltezo; Boehringer Ingelheim GmbH, Ingelheim, Germany), which are biosimilars to adalimumab (Humira; AbbVie Inc. Lake Bluff, IL, USA). The FDA has accepted the concept of extrapolation of indication.

Biosimilar should be prescribed by brand name as well as by international nonproprietary name. It is the responsibility of healthcare professionals to make sure that there is shared decision making by giving all the relevant information and confirm informed consent before initiating biosimilar administration, taking into consideration the preference of the patient. Since biosimilars are essentially the same molecule, switching to a biosimilar will not prevent immunogenicity to the reference medicine which lead to loss of response and adverse events. 
Scientific and clinical evidence is lacking regarding reverse switching, multiple switching and cross-switching among biosimilars. ${ }^{83}$ There should be a robust pharmacovigilance strategy to protect patients and develop the long-term evidence base required to provide patients and clinicians with the necessary assurances on safety and effectiveness. Biosimilars are likely to be produced beyond infliximab and may help reduce cost of IBD treatment throughout the Asia-Pacific region.

\section{Part C: How to Monitor Response-Clinical, Endoscopy, Biomarkers and Mucosal Healing}

\begin{abstract}
Statement 11
Monitoring of disease activity must be performed regularly, including clinical parameters, blood/fecal biomarkers and endoscopy. Cross sectional imaging and capsule endoscopy may be performed as appropriate.

- Level of agreement: (a) 95\%, (b) 5\%, (c) 0\%, (d) 0\%, (e) $0 \%$. - Quality of evidence: II-2

- Classification of recommendation: A
\end{abstract}

The target of IBD treatment includes the resolution of symptoms and intestinal inflammation; therefore, regular monitoring of inflammatory activity is essential for the optimization of IBD treatment. ${ }^{84}$ Clinical response to treatment should be assessed 3-monthly ${ }^{84}$ based on symptoms such as abdominal pain and diarrhea in patients with CD or rectal bleeding and bowel habit in patients with $\mathrm{UC}^{84,85}$ or by using clinical disease activity scores ${ }^{84,86}$ such as $\mathrm{CDAI}^{87,88}$ or Harvey-Bradshaw in$\mathrm{dex}^{89}$ in CD and the partial Mayo Clinic index, ${ }^{90,91}$ simple clinical colitis activity index in UC. The resolution of intestinal inflammation or mucosal healing should be monitored given that mucosal healing is associated with improved outcomes in the need for corticosteroids, hospitalization, sustained clinical remission, and decreased need for surgery in $\mathrm{CD}^{92-94}$ and UC. ${ }^{92,95}$ Clinical symptoms does not necessarily correlate with mucosal healing. ${ }^{96,97}$ Endoscopic assessment, therefore, should be performed after starting treatment 6 to 9 months in $\mathrm{CD}$ and 3 to 6 months in UC. ${ }^{84} \mathrm{CRP}$ and fecal calprotectin are not a target in IBD treatment but may guide objective assessment of disease activity. The normalization of CRP and/or fecal calprotectin at week 10 to 14 of treatment is associated with improved outcomes of clinical remission and mucosal healing in $\mathrm{CD}^{98-101}$ and UC. ${ }^{100,102,103}$ Cross-sectional imaging, to date, is not formally considered treatment target in $\mathrm{UC}^{84,104}$ but play a role in the assessment of CD lesions beyond the reach of endoscopy. ${ }^{84,86}$
The clinical response in CDs may not correlate with small bowel mucosal healing due the disconnect between small bowel mucosal inflammation despite clinical remission. ${ }^{105-109} \mathrm{Im}$ provement of inflammation in one location may not parallel improvement in other sites. ${ }^{110}$ Therefore, the assessment of small bowel mucosal healing in areas that are beyond the reach of esophagogastroduodenoscopy and ileocolonoscopy should be considered as well. Video capsule endoscopy is effective for assessment of small bowel mucosal healing ${ }^{111}$ and may be superior to magnetic resonance enterography in the evaluation of proximal small bowel lesions. ${ }^{112}$ The Lewis score and Capsule Endoscopy CDAI have been validated for the assessment of disease activity and mucosal healing using video capsule endoscopy. ${ }^{113,114}$ However, the price of video capsule endoscopy is high, so it may be more cost-effective in high risk patients such as those who have had multiple small bowel resections or with aggressive small bowel disease. ${ }^{115}$

Capsule retention rate was as high as $13 \%$ in patients with established CD, but only $1.6 \%$ in patients with suspected CD. ${ }^{116}$ Capsule retention rate in the latter group without obstructive symptoms, history of small bowel resection or known stenosis had been reported to be comparable to patients with obscure GI bleeding. ${ }^{117-119}$ Small bowel patency capsule prior to capsule endoscopy, therefore, is not considered necessary in most CD patients. There is a strong correlation in severity and extent of disease between the colon capsule endoscopy and conventional colonoscopy in patients with UC. ${ }^{120,121}$ However, colon capsule endoscopy may underestimate both severity and extent of disease. ${ }^{122,123}$ Colon capsule endoscopy has several limitations including inability to obtain biopsy specimens, chance of incomplete colon capsule endoscopy examination and need for more thorough bowel cleansing preparation. ${ }^{124}$ As a result, colon capsule endoscopy cannot substitute colonoscopy for monitoring of disease activity in patients with UC.

\section{Part D: Withdrawing Immunomodulators/Anti-TNF Drugs in IBD}

\section{Statement 12}

In selected CD and UC patients on combination therapy who have absence of surgery history or fistula, normal CRP and fecal calprotectin level, anti-TNF therapy withdrawal while continuing immunomodulator can be considered if the patient is in clinical and endoscopic remission.

- Level of agreement: (a) 76\%, (b) 19\%, (c) 0\%, (d) 5\%, (e) $0 \%$. - Quality of evidence: II-1

. Classification of recommendation: B 
Little is known about the optimal duration of anti-TNF therapy and/or an immunomodulator for patients with IBD but there are emerging data to guide us. Louis et al. ${ }^{47}$ (STORI) evaluated 115 CD patients in remission for at least 6 months on dual treatment with infliximab and azathioprine. Infliximab was stopped and patients were followed for 1 year whereby, $39 \%$ of patients relapsed despite continuing azathioprine. Response was regained in $88 \%$ patients when infliximab was restarted. Patients with a low risk of relapse can be identified using a combination of clinical and biologic markers including low CRP and fecal calprotectin level and absence of surgical resection. In a follow-up of the STORI cohort up to 7 years, $20 \%$ of the patients who did not restart infliximab or another biologic agent and did not develop major complications and $70 \%$ of these patients had no failure resulting from the de-escalation strategy. ${ }^{125}$ Maintenance of immunomodulator treatment after anti-TNF discontinuation was associated with reduced risk of relapse. Patients with perianal fistulas with good response to anti-TNF therapy have a higher risk of relapse on stopping compared with luminal CD, hence anti-TNF discontinuation is not generally recommended in this population. ${ }^{126}$

In adult UC patients, stopping anti-TNF therapy resulted in relapse rates of $14 \%$ to $42 \%$ at 12 months and $25 \%$ to $47 \%$ at 24 months. Relapse rate were lower in studies which included mucosal healing as part of the definition of remission. In a recent meta-analysis, approximately $50 \%$ of patients who discontinued anti-TNF agents after combination therapy-maintained remission 2 years later but the proportion in remission reduced over time. Importantly, resuming the same anti-TNF in patients who relapse following anti-TNF withdrawal for sustained remission is usually safe and effective. Markers of disease activity, poor prognostic factors, and complicated disease course were associated with increased relapse. ${ }^{46}$

\section{Statement 13}

The decision to withdraw a drug should be made for each individual based on patient preference, disease activity markers, risk of relapse, safety, and cost.

- Level of agreement: (a) 95\%, (b) 5\%, (c) 0\%, (d) 0\%, (e) $0 \%$. - Quality of evidence: II-1

- Classification of recommendation: A

It is important to individualize therapy in patients with IBD. Although effective, anti-TNF agents are expensive and may cause severe adverse event, such as infection and malignancy. Decision analysis models have established that anti-TNF agents are cost-effective in the short term but data are unclear for long term. In Asia, special considerations are required for stopping anti-TNF therapy due to cost, economic burden, high prevalence of infections especially TB, and availability of alternative drugs. When considering stopping drugs in IBD, markers of disease activity, prognostic factors, and past history of disease course or relapse need to be taken into consideration. ${ }^{46}$ Subjects with complex IBD, endoscopically active disease, short disease duration, post-surgical and elevated CRP probably should not stop anti-TNF as their risk of relapse is high and consequence of chronic disease activity likely to result in intestinal damage. In contrast, patients in clinical, biochemical, and endoscopic remission are more likely to remain well when anti-TNF or immunomodulators are stopped. Reintroduction of the same treatment is usually, but not always, successful and close clinical monitoring is required upon any treatment withdrawal. The decision on treatment withdrawal is also based on patient preference. Patients with subclinical disease activity are at much higher risk of relapse when any treatment is reduced or withdrawn. Before withdrawal of any maintenance IBD therapy, re-evaluation of disease activity using a combination of clinical, biochemical, endoscopic/histological, and/or radiological techniques should be performed to assess risks and benefits of stopping. ${ }^{126}$ In developing or newly industrialized countries which lack reimbursement of biologic agents, cost may be an issue which leads to treatment withdrawal. Therefore, discontinuation of therapy needs to be personalized on a case-by-case basis.

\section{Statement 14}

Stopping azathioprine or mercaptopurine mono-therapy in patients with CD and UC is associated with a high risk of relapse and should not be encouraged unless the patient has been in clinical remission for more than 4 years.

. Level of agreement: (a) 48\%, (b) 48\%, (c) 4\%, (d) 0\%, (e) $0 \%$. - Quality of evidence: I

- Classification of recommendation: A

In CD, a multicenter double-blind study of azathioprinetreated patients, in clinical remission for over 3 years found that cumulative risk of relapse after withdrawal at 1, 3, and 5 years was $14 \%, 53 \%$, and $63 \%$, respectively. ${ }^{127}$ Several subsequent controlled trials also showed higher relapse rates in the drug withdrawal arm, from $8 \%$ to $25 \%$ at 6 months, $17 \%$ to $53 \%$ at 12 months, $21 \%$ to $31 \%$ at 18 months, and $31 \%$ at 24 months. ${ }^{126,128}$ In UC, there are fewer studies of stopping immunomodulator 
monotherapy. For UC patients in short-term remission with azathioprine, 1-year relapse rates was seen in $59 \%$ with azathioprine withdrawal. ${ }^{129}$ Overall in both CD and UC, there is high cumulative risk of relapse overtime after withdrawal of immunomodulator monotherapy and it is estimated that approximately one third of patients relapse by 2 years and half to three quarters relapse by 5 years. ${ }^{126,127,130,131}$

It is therefore important to consider in conjunction with the patient, the risks and benefits of continued immunomodulator monotherapy for IBD patients treated for 3 to 4 years if there is no evidence of continuing disease activity. ${ }^{132}$ Factors predictive of relapse following withdrawal of immunomodulator monotherapy include raised markers of subclinical disease activity and disease extent/localization such as peri-anal disease in CD or extensive disease in UC. ${ }^{46}$ Consistent factors associated with disease relapse in $\mathrm{CD}$ after stopping immunomodulators included high CRP, low hemoglobin levels, and increased leukocyte count whereas for UC these included increased leucocyte count, number of relapses on azathioprine and shortened duration on azathioprine. The BERENICE study modeled mortality risk in CD patients according to immunomodulator use, age, and disease extent and favored sustained immunomodulator treatment in $\mathrm{CD}$ patients with extensive colitis, irrespective of age. ${ }^{133}$

\section{Statement 15}

In selected CD and UC patients with absence of surgery or fistula, normal CRP, normal fecal calprotectin level and endoscopic healing withdrawing of biologic monotherapy can be considered if the patient is in clinical remission for more than 4 years.

- Level of agreement: (a) 26\%, (b) 37\%, (c) 37\%, (d) 0\%, (e) $0 \%$. - Quality of evidence: II-3

- Classification of recommendation: B

There remains a lack of high quality studies on stopping monotherapy for anti-TNF in patients with IBD. Such patients usually require anti-TNF or biologic drugs because of previous poor disease control, therefore stopping anti-TNF therapy completely might not be appropriate. More studies are needed ideally randomized controlled trials, to compare the antiTNF discontinuation strategy with a control group where the anti-TNF is maintained, in those with different disease course or those who started therapy at different disease time point. It is possible that early treatment resulting in deep mucosal healing may allow ceasing therapy in selected subjects. Thus, this statement has been rejected.

\section{Part E: Pharmacogenetics for TPMT and NUTD15}

\section{Statement 16}

TPMT testing prior to thiopurine commencement is of limited value in Asian populations. Hence routine measurement is not recommended.

. Level of agreement: (a) 71\%, (b) 29\%, (c) 0\%, (d) 0\%, (e) $0 \%$. - Quality of evidence: II-3

- Classification of recommendation: $\mathrm{C}$

Thiopurines undergo complex metabolism that leads to the formation of the active and potentially myelotoxic metabolite, 6-thioguanine, as well as inactive and potentially hepatotoxic metabolite, 6-methyl mercaptopurine. Two genes are known to be associated with leukopenia in patients on thiopurines; TPMT and NUTD15. The gene encoding TPMT is polymorphic, leading to a large variation in enzyme activity between individuals. Both genotyping and phenotyping studies have shown ethnic variations in gene sequencing and enzyme activity worldwide, with at least 29 mutations in the TPMT gene identified to date. ${ }^{134}$

Low TPMT enzyme activity leads to increased conversion of thiopurines to 6-thioguanine via the hypoxanthine phosphoribosyltransferase pathway. Minimal TPMT activity can cause early, potentially life-threatening myelosuppression in the setting of thiopurine use. Epidemiological studies in the Caucasian population have shown a trimodal distribution of TPMT enzyme activity, with 89\% having normal or high activity, $11 \%$ having intermediate activity and $0.3 \%$ having minimal activity. ${ }^{135}$ The utility TPMT testing in personalizing thiopurine treatment has remained controversial in the Asian population. TPMT variants are generally rare among Asian populations. ${ }^{136}$ Interestingly, although the frequency of TPMT mutations is lower in the Asians compared to the Caucasians ( $\sim 3 \%$ vs. 10\%), the frequency at which thiopurine-induced leukopenia occurs in Asians is paradoxically considerably higher; $5 \%$ in Caucasians compared to $35.4 \%$ in Korean, ${ }^{137} 15.8 \%$ in Japanese, ${ }^{138}$ and $18.1 \%$ in Chinese. ${ }^{139}$ The thiopurine dose given in these group of patients were also much less than the recommended weight-based dosing. Hence, more frequent and severe leukopenia is expected with standard doses of thiopurines in this population. This suggests that TPMT genotyping or phenotyping does not seem to be very useful in Asian population and also highlights the existence of other underlying race specific 
genetic polymorphisms in thiopurine response.

\section{Statement 17}

In the Asian population, NUDT15 genotyping, prior to thiopurine commencement is recommended if available.

- Level of agreement: (a) 89\%, (b) $11 \%$, (c) $0 \%$, (d) $0 \%$, (e) $0 \%$. - Quality of evidence: II-2

- Classification of recommendation: B

The NUDT15 gene codes for an enzyme in the regulation of 6-thioguanine concentrations by converting 6-thioguanosine triphosphate to 6-thioguanosine monophosphate. ${ }^{140}$ Recent genome-wide association studies described a missense variant in the NUDT15 gene rs116855232 (c.415C > T or p.Arg139Cys variant) that is strongly associated with thiopurine-related myelosuppression in patients with $\mathrm{IBD}^{4}$ and in children with acute lymphoblastic leukemia (ALL). ${ }^{141}$ NUDT15 genetic variation is substantially overrepresented in Asians and is their predominant genetic cause for thiopurine toxicity. ${ }^{137,141}$ The NUDT15 risk allele encoding p.Arg139Cys is more common in Asians than in Caucasians, with reported allele frequencies of $10.4 \%$ in Koreans, $7 \%$ in Japanese, $13 \%$ in Chinese and $2 \%$ in an admixed American population. ${ }^{137}$

Yang et al. ${ }^{137}$ identified the NUDT15 variant (p.Arg139Cys) as a significant risk factor for thiopurine-induced early leukopenia in Korean CD patients. The $978 \mathrm{CD}$ patients were included in the study. The p.Arg139Cys risk variant was present in $89.4 \%$ of cases (59/66) developing early leukopenia but in only $6.8 \%$ of controls (43/632). This NUDT15 allele had a sensitivity of $89.4 \%$ (59/66), specificity of $93.2 \%$ (589/632) and an area under the curve value of 0.92 for early leukopenia. In addition, there was a positive gene dose effect of the NUDT15 risk allele in development of thiopurine associated leucopenia. As the number of copies of the NUDT15 risk allele increased, the dose of thiopurines at which leukopenia occurred decreased, the interval from the onset of thiopurine therapy to the development of leukopenia decreased and the grade of the observed leukopenia increased.

The results of this study were reproduced in a study of 663 children with ALL. ${ }^{141}$ The NUDT15 variant was absent from the African population, uncommon in Europeans and it was the most prevalent in East Asians and Hispanics. These NUDT15 variants are highly penetrant. Patients with the TT genotype at rs116855232 (homozygous for the risk allele at p.Arg139Cys) were very sensitive to mercaptopurine, with an average dose intensity of $8.3 \%$, compared with patients with TC and CC ge-
Table 2. The Genotype and Allele Frequencies of NUDT15 c.415C>T in Different Ethnicity ${ }^{133}$

\begin{tabular}{lrrrc}
\hline & \multicolumn{4}{c}{ Ethnicity (\%) } \\
\cline { 2 - 5 } & Asian & Hispanic & European & African \\
\hline Genotype NTUD15 c.415C $>$ T & & & \\
CC & 75.84 & 92.34 & 99.51 & 100.00 \\
CT & 21.76 & 7.21 & 0.49 & 0 \\
T & 2.39 & 0.45 & 0 & 0 \\
Allele & & & & \\
C & 86.72 & 95.95 & 99.76 & 100.00 \\
T & 13.28 & 4.05 & 0.24 & 0 \\
\hline
\end{tabular}

notypes, who tolerated $63 \%$ and $83.5 \%$ of the planned dose, respectively.

Such association has been demonstrated by multiple independent studies. Recently published systemic review and meta-analysis, included 7 studies with total of 1,138 patients with IBD or ALL, of which 311 patients carried the NUDT15 415T allele. ${ }^{142}$ This meta-analysis provided evidence that $\mathrm{T}$ carriers of this NUDT15 c.415C > T variant were significantly correlated with high incidences of thiopurine-induced leukopenia. This correlation was especially strong in TT patients, where it was found to be significantly increased by 6.54 -fold. They also found that the NUDT15 c.415C > T variant was common in Asians and Hispanics, but rare in Europeans and Africans; the frequency of the NUDT15 c.415C > T distribution varied substantially by race/ethnicity (Table 2 ).

This strongly indicates the potential of NUDT15 genotype, particularly c.415C $>\mathrm{T}$ (rs116855232) variant, to guide individualized thiopurine dosing to mitigate toxicity, with the same principle used for TPMT based dose adjustments, especially in the Asian population. Therefore, integration of NUDT15 analysis in thiopurine dosing algorithm may have a major implication for Asian populations, whereas TPMT variants are most informative for thiopurine toxicity in Europeans and Africans. A dosing algorithm that incorporates NUDT15 variants would potentially provide a robust approach to personalize thiopurine therapy in the Asian population.

\section{Statement 18}

In patients with CD and UC we recommend a steady state trough infliximab level between 3 and $7 \mu \mathrm{g} / \mathrm{mL}$ and adalimumab trough level between 4 and $8 \mu \mathrm{g} / \mathrm{mL}$.

- Level of agreement: (a) 45\%, (b) 41\%, (c) 14\%, (d) 0\%, (e) $0 \%$. - Quality of evidence: II-2

- Classification of recommendation: B 
Anti-TNF drug concentrations and of antidrug antibodies may help to correlate with clinical outcomes. Studies have proposed several different optimal cutoff levels. This variability is explained by multiple factors: (1) the heterogeneity of the available assays. Different assays have been used and they are not necessarily equivalent making extrapolation difficult; and (2) the diversity of studies outcomes. The studies used to derive different target trough concentrations were studies of patients on maintenance therapy in various stages of response or remission.

Table 3 summarizes the potential target infliximab trough drug level from the existing literature to predict clinical disease activity. ${ }^{99,143-149}$ According to the studies, infliximab trough $>3$ $\mu \mathrm{g} / \mathrm{mL}$ is predictive of failure to respond to dose escalation, and also remission rates appear to plateau for infliximab trough levels above $7 \mu \mathrm{g} / \mathrm{mL}$. Based on the currently available evidence, we suggest target trough concentrations of 3-7 $\mu \mathrm{g} / \mathrm{mL}$ for infliximab.

Table 4 summarizes the potential target adalimumab level from the existing literature to predict clinical disease activity. ${ }^{150-154}$ For adalimumab, a trough greater than $4 \mu \mathrm{g} / \mathrm{mL}$ was predictive of nonresponse to dose escalation, and remission rates plateau above $8 \mu \mathrm{g} / \mathrm{mL}$. The upper limit of the therapeu- tic range for adalimumab is less well defined compared to infliximab. Based on the currently available evidence, we suggest target trough concentrations of $4-8 \mu \mathrm{g} / \mathrm{mL}$ for adalimumab. For patients who are not in clinical remission and have limited therapeutic options beyond the anti-TNF agent they are on, we recommend aiming for therapeutic levels in the higher end of the quoted therapeutic range.

\section{Statement 19}

Trough levels higher than the standard range may be appropriate for those with fistulizing $\mathrm{CD}$ or in aiming for mucosal healing.

- Level of agreement: (a) 45\%, (b) 41\%, (c) 14\%, (d) $0 \%$, (e) $0 \%$. - Quality of evidence: II-3

- Classification of recommendation: $\mathrm{C}$

A different target therapeutic range for infliximab or adalimumab may be appropriate for specific treatment end-points and/or disease phenotypes. Higher trough levels are often needed to achieve endoscopic remission or mucosal healing and closure of fistulas in CD. In a retrospective cross-sectional study of 145 IBD patients treated with infliximab or adalimumab, for endoscopic remission, an optimal infliximab trough level ap-

Table 3. Target Infliximab trough Drug Level from Various Studies

\begin{tabular}{lcccccc}
\hline Study & Year & Disease & Design & No. of subjects & Assay & Threshold $(\mu \mathrm{g} / \mathrm{mL})$ \\
\hline Steenholdt et al. $^{143}$ & 2011 & IBD & Obs & 106 & RIA & $\geq 2.8$ \\
${\text { Bortlik et al. }{ }^{144}}^{9}$ & 2013 & CD & Obs & 84 & ELISA & $\geq 3.0$ \\
Cornillie et al. $^{99}$ & 2014 & CD & RCT & 144 & ELISA & $\geq 3.5$ \\
Adedokun et al. $^{145}$ & 2014 & UC & RCT & 728 & ELISA & $\geq 3.7$ \\
Levesque et al. $^{146}$ & 2014 & CD & Obs & 327 & HMSA & $\geq 3.0$ \\
Vande Casteele et al. ${ }^{147}$ & 2015 & CD & Obs & 483 & HMSA & $\geq 2.8$ \\
Reinisch et al. $^{148}$ & 2015 & CD & RCT & 203 & ELISA & $\geq 3.0$ \\
Vande Casteele et al. & ${ }^{149}$ & IBD & RCT & 263 & ELISA & $\geq 3.7$ \\
\hline
\end{tabular}

Obs, observational; RIA, radioimmunoassay; RCT, randomized controlled trail; HMSA, homogeneous mobility shift assay.

Table 4. Target Adalimumab trough Drug Level from Various Studies

\begin{tabular}{|c|c|c|c|c|c|c|}
\hline Study & Year & Disease & Design & No. of subjects & Assay & Threshold $(\mu \mathrm{g} / \mathrm{mL})$ \\
\hline Karmiris et al. ${ }^{150}$ & 2009 & $C D$ & Prospective & 168 & ELISA & $6.2-8.9$ \\
\hline Roblin et al. ${ }^{151}$ & 2014 & IBD & Cross sectional & 40 & ELISA & $>4.9$ \\
\hline Mazor et al. ${ }^{152}$ & 2014 & $C D$ & Cross sectional & 71 & ELISA & 5.85 \\
\hline Roblin et al. ${ }^{153}$ & 2014 & $\mathrm{IBD}$ & Prospective & 82 & ELISA & 4.9 \\
\hline Morita et al. ${ }^{154}$ & 2016 & $C D$ & Retrospective & 42 & ELISA & $5.57-7.9$ \\
\hline
\end{tabular}


pears to be $6-10 \mu \mathrm{g} / \mathrm{mL}$, while an optimal adalimumab trough appears to be $8-12 \mu \mathrm{g} / \mathrm{mL}^{155}$

Studies have shown that a higher infliximab trough level is required to heal perianal fistulizing $\mathrm{CD}$. A retrospective crosssectional study looking at $117 \mathrm{CD}$ patients with perianal fistula treated with infliximab for at least 24 weeks, assessed the correlation between perianal fistula healing and trough levels of infliximab. ${ }^{31}$ There was a linear relationship between quartiles and fistula healing when infliximab levels were stratified by quartiles. Infliximab trough level cutoffs above 2.9, 10.1, and $20.2 \mu \mathrm{g} / \mathrm{mL}$ were associated with fistula healing rates of $65 \%$, $79 \%$ and $86 \%$, respectively. In the multivariate analysis, only infliximab level $\geq 10.1 \mu \mathrm{g} / \mathrm{mL}$ maintained statistical significance for fistula healing. Data on adalimumab levels for healing in fistulizing CD is lacking. Based on the same study, in the context of mucosal healing, when infliximab levels were stratified by quartiles, there was again a linear relationship between quartiles and mucosal healing. ${ }^{31}$ Infliximab trough level cutoffs above $2.9,10.1$, and $20.2 \mu \mathrm{g} / \mathrm{mL}$ were associated with mucosal healing rates of $65 \%, 79 \%$ and $86 \%$, respectively. In the multivariate analysis, infliximab level $<10 \mu \mathrm{g} / \mathrm{mL}$ was independently associated with lack of mucosal healing. These studies suggest that higher infliximab levels are associated with fistula and mucosal healing. Drug levels higher than what has been described for clinical remission may be needed to achieve mucosal healing and fistula resolution in $\mathrm{CD}$. In a retrospective cross-sectional study of 145 IBD patients treated with infliximab or adalimumab, for endoscopic remission, an optimal infliximab trough level appears to be $6-10 \mu \mathrm{g} / \mathrm{mL}$, while an optimal adalimumab trough appears to be $8-12 \mu \mathrm{g} /$ $\mathrm{mL}{ }^{155}$ Studies have shown that a higher infliximab trough level is required to heal perianal fistulizing $\mathrm{CD}$. A retrospective cross-sectional study looking at $117 \mathrm{CD}$ patients with perianal fistula treated with infliximab for at least 24 weeks, assessed the correlation between perianal fistula healing and trough levels of infliximab. ${ }^{31}$ When infliximab levels were stratified by quartiles, there was a linear relationship between quartiles and fistula healing. Infliximab trough level cutoffs above 2.9, 10.1 , and $20.2 \mu \mathrm{g} / \mathrm{mL}$ were associated with fistula healing rates of $65 \%, 79 \%$, and $86 \%$, respectively. In the multivariate analysis, only infliximab level $\geq 10.1 \mu \mathrm{g} / \mathrm{mL}$ maintained statistical significance for fistula healing. There are few data on adalimumab levels for healing in fistulizing CD.

Based on the same study, in the context of mucosal healing, when infliximab levels were stratified by quartiles, there was again a linear relationship between quartiles and mucosal heal- ing. ${ }^{31}$ Infliximab trough level cutoffs above 2.9, 10.1, and 20.2 $\mu \mathrm{g} / \mathrm{mL}$ were associated with mucosal healing rates of $65 \%$, $79 \%$ and $86 \%$, respectively. In the multivariate analysis, infliximab level $<10 \mu \mathrm{g} / \mathrm{mL}$ was independently associated with lack of mucosal healing. These suggest that infliximab levels are associated fistula and mucosal healing. Drug levels higher than what has been described for clinical remission may be needed in order to achieve therapeutic success in mucosal healing and penetrating $\mathrm{CD}$, even more so in penetrating $\mathrm{CD}$.

These consensus statements favored use of reactive therapeutic drug monitoring (TDM) in patients with active IBD to help guide management. An algorithm of TDM of biologic agent testing in IBD is summarized in Fig. 1. The testing of antidrug antibodies is variable between different commercial assays and there is no standardized reporting of these values. There is, therefore, greater variability in the detection of antidrug antibodies than anti-TNF drug levels between different assays. Low-titer antidrug antibodies may be transient and non-neutralizing. In contrast, high-titer antibodies, especially with undetectable trough drug concentrations, are generally persistent and neutralizing and associated with loss of treatment efficacy. No antidrug antibody cutoffs have been established to date to differentiate high from low antibody titers. An ideal assay is one that has cutoffs that directly correlate against clinical data.

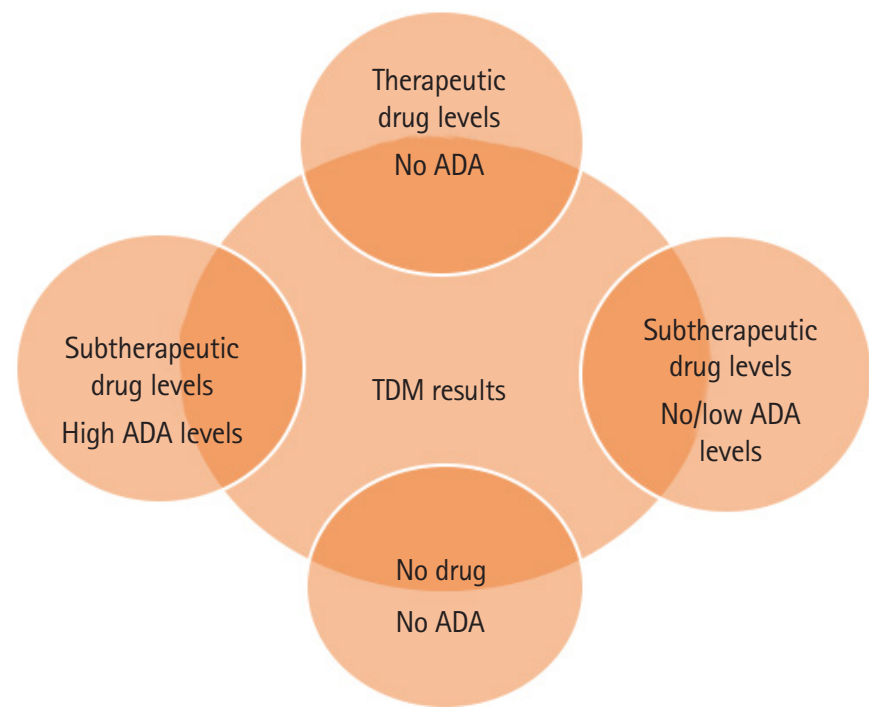

Fig. 1. Possible permutation of therapeutic drug monitoring (TDM) results. ADA, antidrug antibody. 


\begin{abstract}
Statement 20
Patients with active inflammatory disease and therapeutic drug trough levels (suggesting pharmacodynamic failure) should ideally be switched out of class but switch within class may be effective.

- Level of agreement: (a) 33\%, (b) 48\%, (c) 19\%, (d) 0\%, (e) $0 \%$. - Quality of evidence: II-3

- Classification of recommendation: C
\end{abstract}

If drug trough levels were within the therapeutic range, active inflammatory disease needs to be objectively confirmed with endoscopy, histology, imaging and/or fecal calprotectin. Confirmation of active inflammation is indicative of pharmacodynamic failure, suggestive that non-TNF driven inflammatory pathways may predominate and there may not be significant benefit from anti-TNF dose escalation or switching to another anti-TNF agent. Subjects should instead be ideally switched out of class. In a retrospective study of 247 Israeli IBD patients with loss of response to either infliximab or adalimum$a b$, the correlation between the outcomes of different interventions and trough levels of drug or antidrug antibodies during loss of response was evaluated. ${ }^{156}$ In cases with adequate infliximab or adalimumab drug trough levels at the time of loss of response, the clinical efficacy was significantly better after switching out of class than for anti-TNF dose increase or switching to another anti-TNF drug. A small proportion of patients did recapture response by switching within class. In countries with limited availability of biologic agents available, switching within class may be attempted. Alternatively, referral to a specialized IBD center that is recruiting subjects for clinical drug trials can also be recommended.

\footnotetext{
Statement 21

Patients with active inflammatory disease and undetectable drug trough levels and no antidrug antibodies (suggesting nonimmune mediated pharmacokinetic failure) should have adherence checked first followed by anti-TNF dose escalation.

- Level of agreement: (a) 70\%, (b) 30\%, (c) 0\%, (d) 0\%, (e) $0 \%$. - Quality of evidence: II-3

- Classification of recommendation: $\mathrm{C}$
}

In this scenario once noncompliance is excluded, the patient may have nonimmune mediated pharmacokinetic failure. Nonimmune mediated pharmacokinetic failure is due to under-dosing or the anti-TNF agent being rapidly cleared via a mechanism other than antidrug antibodies. Nonimmune mediated pharmacokinetic failure responds better to dose escalation rather than switching within class. Following dose escalation, we recommend repeating TDM once steady state is again achieved.

Subtherapeutic drug levels and negative antidrug antibodies may also occur early in immune mediated pharmacokinetic failure (if low antidrug antibodies titers complex with anti-TNF drugs and are cleared from the circulation). Repeat testing following dose escalation may detect those with early immune-mediated pharmacokinetic failure, as on repeat testing drug levels may become undetectable with detectable antidrug antibodies.

\section{Statement 22}

Patients with active inflammatory disease and undetectable drug trough levels and low titers of antidrug antibodies, which suggests immune mediated pharmacokinetic failure, should have an immunomodulator added or optimized and/or anti-TNF dose escalation.

- Level of agreement: (a) 64\%, (b) 36\%, (c) 0\%, (d) 0\%, (e) $0 \%$. - Quality of evidence: II-3

- Classification of recommendation: B

Low titers of antidrug antibodies may be overcome with the addition of or optimization of an immunomodulator and/or anti-TNF dose escalation. If the patient is not significantly unwell, a stepwise approach may be taken by adding or optimizing an immunomodulator followed by dose escalation if the former fails to achieve remission. If on repeat testing, drug levels are still undetectable with positive antidrug antibodies, regardless of titers, the patient should be treated as per Statement 23.

\section{Statement 23}

Patients with active inflammatory disease and undetectable drug trough levels and high titers of antidrug antibodies suggest immune-mediated pharmacokinetic failure. Options include addition or optimization of an immunomodulator, and/or switching within or out of class.

- Level of agreement: (a) 68\%, (b) 32\%, (c) 0\%, (d) 0\%, (e) $0 \%$. - Quality of evidence: II-2

- Classification of recommendation: B

To understand the rationale behind the recommendations, these are the evidence looking at (1) the outcome of dose in- 
tensification or switch according to antidrug antibodies titers; and (2) the role of immunomodulators on anti-TNF drug level and immunogenicity.

In a retrospective study of 247 IBD patients with loss of response to infliximab or adalimumab, the effect of dose intensification in patients with high-titer antidrug antibody and no/ low-titer antidrug antibody was studied. ${ }^{156}$ There was no difference in the anti-TNF drug level after dose intensification in patients with high-titer antidrug antibodies. In contrast, dose intensification significantly increased anti-TNF drug levels in patients with no/low antidrug antibodies titers. Vande Casteele et al. ${ }^{157}$ evaluated 90 IBD subjects with loss of response to infliximab and found in those with low levels of antibodies against infliximab $(<9.1 \mathrm{U} / \mathrm{mL})$, infliximab dose intensification was able to recapture response. In contrast, patients with sustained levels of antibodies against infliximab of $>9.1 \mathrm{U} / \mathrm{mL}$ had a poorer response to dose intensification.

A prospective study on 82 IBD patients ${ }^{154}$ examined the impact of TDM on loss of response to adalimumab. They found that in those with low antidrug antibody titers against adalimumab, dose intensification was able to recapture response compared to patients with high antidrug antibodies. However, in the group with high level of antidrug antibodies to adalimumab, upon switching to infliximab, they were able to recapture response in a significant proportion of patients. Based on a retrospective study of 247 IBD patients, ${ }^{156}$ in those with loss of response with no/low antidrug antibody levels to either adalimumab or infliximab, dose intensification resulted in a significantly longer duration of recapturing response than switching to another anti-TNF. However, in cases with loss of response with high antidrug antibody levels to either adalimumab or infliximab, switching to another anti-TNF resulted in a significantly longer duration of regained response than dose intensification.

With the conventional thiopurines weight-based dosing regimen $(2.0-2.5 \mathrm{mg} / \mathrm{kg} / \mathrm{day}$ azathioprine and $1.0-1.5 \mathrm{mg} / \mathrm{kg} /$ day 6-mercaptopurine), placebo-controlled studies reported response rates between $42 \%$ and $80 \% .{ }^{158-160}$ Given the variable results using conventional dosing, thiopurine metabolite measurements are increasingly being used to optimize thiopurine therapy in IBD and improve clinical outcomes.

Data suggest that 6-thioguanine nucleotide concentrations in excess of $235 \mathrm{pmol} / 8 \times 10^{8}$ erythrocytes are associated with clinical remission in a significant proportion of patients. ${ }^{161,162}$ The 6-thioguanine nucleotide upper limit is based on studies showing that the proportion of patients in remission does not increase significantly with 6-thioguanine nucleotide concentrations greater than $450 \mathrm{pmol} / 8 \times 10^{8}$ erythrocytes, whereas there is an increased risk of myelotoxicity above this level. ${ }^{161,163,164}$ The dose of thiopurine correlated poorly with 6-thioguanine nucleotide levels $(\mathrm{r}=0.0009) .{ }^{161}$

Dose optimization studies using 6-thioguanine nucleotide levels have also been reported. Two retrospective Australian studies have shown that optimization of thiopurines in patients with subtherapeutic 6-thioguanine nucleotide levels can lead to improvement in clinical outcomes in $88 \%$ and $78 \%$ of patients after dose escalation of thiopurines, respectively. ${ }^{165,166}$ A pivotal metabolite study demonstrated that high levels of 6-methylmercaptopurine were associated with hepatotoxicity with elevated levels of transaminases. The incidence of hepatotoxicity in this study was $17 \%$ with median 6-methylmercaptopurine levels of $5,463 \mathrm{pmol} / 8 \times 10^{8}$ erythrocytes in those patients with abnormal liver function tests compared to 2,213 $\mathrm{pmol} / 8 \times 10^{8}$ erythrocytes in those with normal liver function tests $(P<0.05)$. The risk of hepatotoxicity increased 3 -fold ( 18 vs. $6 \%, P<0.05)$ when 6 -methylmercaptopurine exceeded $5,700 \mathrm{pmol} / 8 \times 10^{8}$ erythrocytes. ${ }^{4}$ There was no correlation of 6-methylmercaptopurine levels with clinical efficacy or thiopurine dose. Hence, the therapeutic range for use in clinical practice for 6-thioguanine nucleotide is $235-450 \mathrm{pmol} / 8 \times 10^{8}$ erythrocytes. For 6-methylmercaptopurine, a level of less than $5,700 \mathrm{pmol} / 8 \times 10^{8}$ erythrocytes mitigate the risk of hepatotoxicity.

Several studies have shown the benefit of using combination therapy with anti-TNFs and immunomodulators. The mechanism to explain the improved efficacy with combination therapy includes higher anti-TNF levels and decreased immunogenicity. There is a significant correlation between anti-TNF drug level and antidrug antibody level, and the use of immunomodulators. ${ }^{167}$ The SONIC study demonstrated concomitant azathioprine usage resulted in higher infliximab trough levels most likely through reduced immunogenicity. ${ }^{34}$ Similarly, in the COMMIT trial, concomitant methotrexate was associated with a higher infliximab trough level and reduced immunogenicity. ${ }^{40}$

Based on a cross-sectional study, higher 6-thioguanine nucleotide levels correlate with higher trough infliximab concentrations in IBD patients on combination therapy. Patients with detectable infliximab antibodies had significantly lower 6-thioguanine nucleotide levels. ${ }^{168}$

Ben-Horin et al. ${ }^{38}$ also showed the benefit of adding immunomodulators to revert immunogenicity and increasing inflix- 


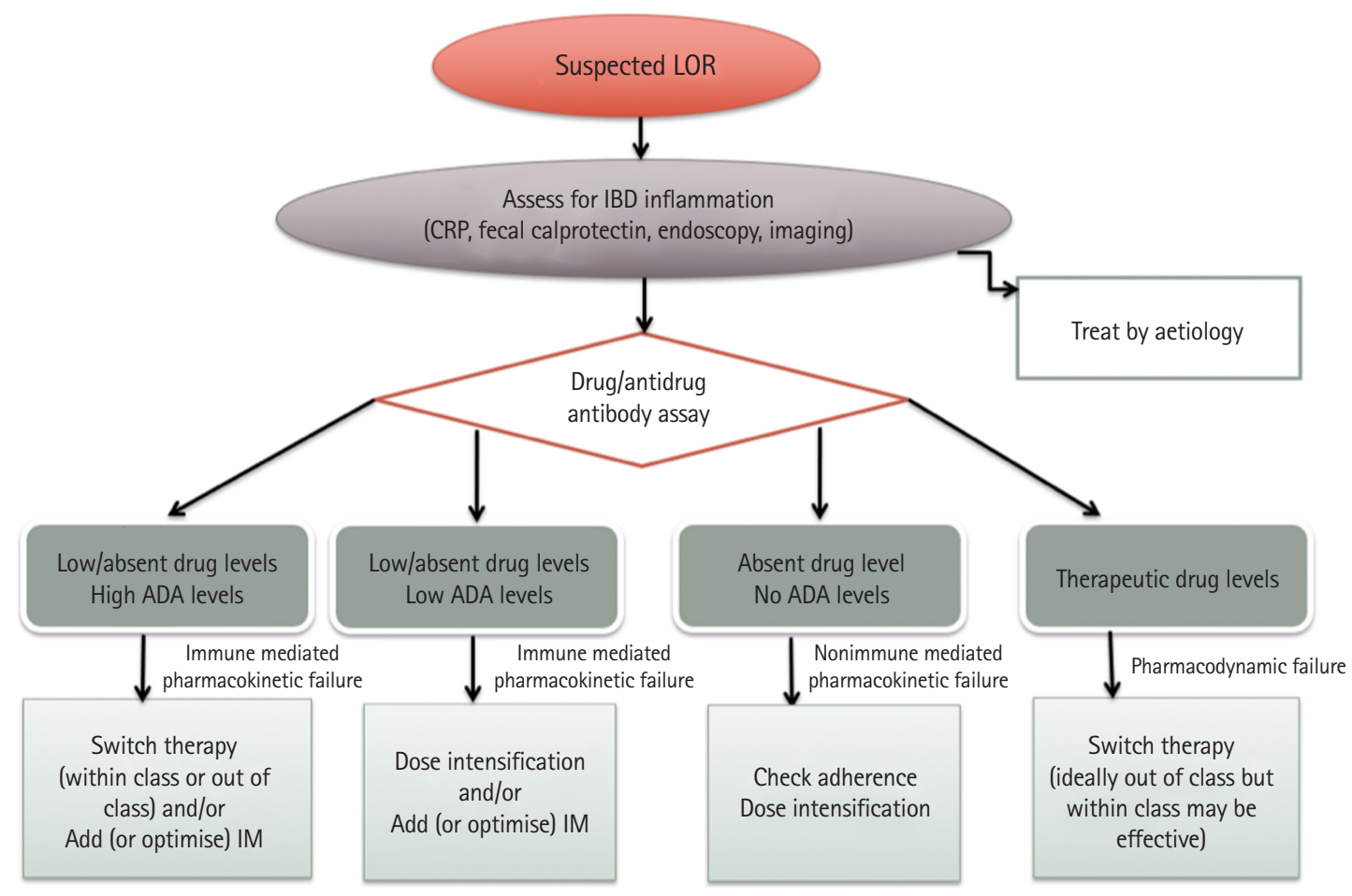

Fig. 2. Summary algorithm to guide management according to therapeutic drug monitoring loss of response (LOR). ADA, antidrug antibody; IM, immunomodulator.

imab levels in patients who developed antidrug antibodies to infliximab with subtherapeutic infliximab drug level. Fig. 2 summarizes the recommendation on the interpretation of TDM for anti-TNF to guide management in patients with loss of response.

\section{Statement 24 \\ HBsAg, hepatitis B surface antibody (HBsAb), and hepatitis B core antibody (HBcAb) should be tested routinely before initiation of systemic corticosteroids, immunomodulators and biologic agents. \\ - Level of agreement: (a)100\%, (b) 0\%, (c) 0\%, (d) 0\%, (e) $0 \%$. - Quality of evidence: II-3 \\ - Classification of recommendation: B}

In Asia, $\mathrm{HBV}$ infection is endemic, and East Asia, where over $8 \%$ of males over the age of 35 are positive for the HBsAg, has the highest prevalence of all Asian regions. ${ }^{169}$ To minimize the risk of reactivation of the virus, immunosuppressive therapy should proceed only after screening in order to avoid life threatening situations. ${ }^{170,171} \mathrm{HBV}$ vaccination is recommended in patients who are negative for $\mathrm{HBsAg}, \mathrm{HBsAb}$, and $\mathrm{HBcAb}$.

\section{Statement 25}

In patients who are HBsAg and/or HBcAb positive, HBV DNA quantification is recommended before the initiation of systemic corticosteroids, immunomodulators and biologic agents.

- Level of agreement: (a) 77\%, (b) 23\%, (c) 0\%, (d) 0\%, (e) $0 \%$. - Quality of evidence: II-3

- Classification of recommendation: $\mathrm{C}$

In patients with IBD, $25 \%$ to $36 \%$ of those who are HBsAg positive experienced liver dysfunction. ${ }^{172,173}$ Most cases of HBV reactivation have been observed in HBV-infected IBD patients treated with 2 or more immunomodulators for a long period of time, were positive for HBV DNA, and/or had not received prophylactic antiviral treatment. ${ }^{171}$ Therefore, we recommend checking the HBV DNA titer before initiating systemic corticosteroids, immunomodulators and biologic agents.

\section{Statement 26}

Antiviral treatment for prophylaxis of $\mathrm{HBV}$ reactivation is recommended in patients with detectable HBV DNA, before initiation of systemic corticosteroids, immunomodulators and biologics.

- Level of agreement: (a) 82\%, (b) 14\%, (c) 4\%, (d) 0\%, (e) $0 \%$. - Quality of evidence: II-2

- Classification of recommendation: A 
Patients with no antiviral therapy should be monitored closely and antiviral treatment initiated when there is increase in HBV DNA titer. It is recommended for patients with detectable HBV DNA to undergo antiviral prophylaxis using nucleotide/nucleoside analogues. The treatment should start 2 weeks before the commencement of immunomodulators. Prophylaxis should continue for 6 to 12 months after discontinuation of immunomodulators. Entecavir and tenofovir have a rapid onset of action, high antiviral potency, and low incidence of resistance, and are preferred in patients with IBD. ${ }^{171}$

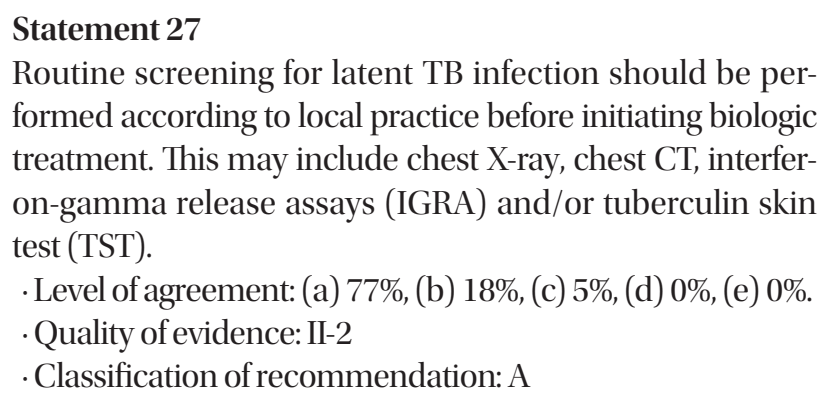

Due to the use of immunosuppressive therapy, patients with IBD are at higher risk of active TB infection than the general population. ${ }^{171}$ Extrapulmonary TB is more common in patients on immunosuppressive agents, compared to the general population ( $30 \%$ vs. $13 \%$, in Taiwan, respectively). ${ }^{174-176}$ Atypical presentation and disseminated disease is also more common in patients treated with anti-TNF, making diagnosis more difficult. ${ }^{171}$ In Taiwan, 5.8\% of cases of extrapulmonary $\mathrm{TB}$ were reported to have occurred in the GI tract. ${ }^{177}$ Screening for latent TB should be performed in all CD patients prior to biologic therapy through physical examination, chest radiography, and TST or IGRA (QuantiFERON-TB GOLD). It should be noted that results of TST is affected by prior BCG vaccination, whereas IGRA is unaffected by prior BCG exposure. ${ }^{178}$

\begin{abstract}
Statement 28
In patients diagnosed with latent $\mathrm{TB}$, prophylactic treatment for the prevention of TB reactivation is effective, therefore, chemoprophylaxis should be started (preferably 3-4 weeks) before the use of biologic agents.

- Level of agreement: (a) 77\%, (b) 23\%, (c) 0\%, (d) 0\%, (e) $0 \%$. - Quality of evidence: II-3

- Classification of recommendation: A
\end{abstract}

Chemoprophylaxis is highly effectively in decreasing the risk of $\mathrm{TB}$ reactivation in patients with latent $\mathrm{TB}$ receiving the anti-TNF treatment. ${ }^{179}$ Treatment of latent TB should follow the current local guideline recommendations. ${ }^{171,177}$ Although there is no robust data, European Crohn's and Colitis Organisation ${ }^{171}$ as well as Asian Organization for Crohn's and Colitis (AOCC)/Asia Pacific Association of Gastroenterology (APAGE) ${ }^{180}$ guidelines and most authorities suggest that it should be safe to start the biologic agents (at least 3-4 weeks) after the initiation of anti-TB drugs according to the clinical experience and observational study. ${ }^{181,182}$ Consultation with an infectious disease or chest specialist for multidisciplinary care is recommended. ${ }^{171,177}$

\section{Statement 29}

During biologic therapy, patients should be monitored for contact history, symptoms and signs of active TB. Regular chest X-ray and IGRA may be considered.

- Level of agreement: (a) 68\%, (b) 32\%, (c) 0\%, (d) 0\%, (e) $0 \%$. - Quality of evidence: III

- Classification of recommendation: B

Patients with IBD receiving biologic treatment should be monitored regularly for signs and symptoms of active TB disease. Chest radiography and IGRA should be performed ideally every 6 months, or at least annually in clinical practice for IGRA. ${ }^{177}$ Travel and TB contact history should be monitored while the patient is receiving immunosuppressive treatment An infectious disease specialist should be consulted when necessary.

\section{CONCLUSIONS}

The use of immunomodulators and biologic agents for the management of IBD is increasingly common. The data demonstrating the safety, efficacy of biologic agents such as infliximab, adalimumab and their corresponding biosimilars has been reviewed and discussed in this paper. We have also described strategies for initiation and de-escalation when considering the administration of these drugs in Asia. Special consideration must be paid to the risk of under-treatment due to financial constraints leading to episodic treatment resulting in suboptimal disease control. We have also highlighted HBV and TB infections, which may complicate the use of biologic agents and immunomodulators. In administering immunomodulators, the data suggest that clinicians take into account the pharmacogenetics of NUTD15 in the Asian population 
whereas the pharmacogenetics of TMPT are not as relevant.

The use of immunomodulators and biologic agents for the management of IBD is increasingly common in Asia. The data demonstrating the safety and efficacy of biologic agents and their corresponding biosimilars has been summarized in these consensus statements. We have also described strategies for the initiation and de-escalation of these drugs. In Asia, undertreatment of IBD is possible due to financial constraints resulting in episodic treatment with the risk of sub-optimal disease control. We also highlighted HBV and TB infections that may complicate the use of biologic agents and immunomodulators. In administering immunomodulators, the data suggest that clinicians take into account the pharmacogenetics of NUDT15 in the Asian population whereas the pharmacogenetics of TMPT are not as relevant.

The Asia Pacific Association of Gastroenterology (APAGE) Working Group on IBD in collaboration with AOCC has endeavored to present through this review, a comprehensive and authoritative understanding of the best practices for treating UC and CD with immunomodulators and biologic agents. The consensus statements have been developed through a rigorous process according to the modified Delphi system and has been formulated with input from various experts in the region that comprise the APAGE and AOCC. These consensus statements should be read with previous consensus statements on the definition and management of IBD in previous reviews. It is hoped that these recommendations may not only guide clinicians in the best-use of biologic agents and immunomodulators but also be used for hospital and national regulatory authorities to accept these drugs onto the pharmacy formulary or for reimbursement.

\section{FINANCIAL SUPPORT}

Unrestricted educational grants were obtained from Medtronic, Takeda, LF Asia, Journal of Gastroenterology and Hepatology Foundation and Asian Pacific Association of Gastroenterology.

\section{CONFLICT OF INTEREST}

No potential conflict of interest relevant to this article was reported.

\section{AUTHOR CONTRIBUTION}

Conceptualization: Ooi CJ, Hilmi I, Banerjee R, Leong RW.
Methodology: Ooi CJ, Hilmi I, Banerjee R, Leong RW. Formal analysis: Ooi CJ, Hilmi I. Funding acquisition: Ooi CJ. Project administration: Ooi CJ, Hilmi I. Visualization: Ooi CJ, Hilmi I. Writing - original draft: Ooi CJ, Hilmi I, Banerjee R, Chuah SW, Ng SC, Wei SC, Makharia GK, Pisespongsa P, Ahuja V, Leong RW. Writing - review and editing: Ooi CJ, Hilmi I, Banerjee R, Chuah SW, Ng SC, Wei SC, Leong RW. Approval of final manuscript: all authors.

\section{REFERENCES}

1. Ooi CJ, Makharia GK, Hilmi I, et al. Asia Pacific Consensus Statements on Crohn's disease. Part 1: definition, diagnosis, and epidemiology: (Asia Pacific Crohn's Disease ConsensusPart 1). J Gastroenterol Hepatol 2016;31:45-55.

2. Ooi CJ, Makharia GK, Hilmi I, et al. Asia-Pacific consensus statements on Crohn's disease. Part 2: management. J Gastroenterol Hepatol 2016;31:56-68.

3. Linstone HA, Turoff M. The Delphi method: techniques and applications. Boston: Addison-Wesley, 1975.

4. The periodic health examination. Canadian Task Force on the Periodic Health Examination. Can Med Assoc J 1979;121: 1193-1254.

5. Renna S, Cottone M, Orlando A. Optimization of the treatment with immunosuppressants and biologics in inflammatory bowel disease. World J Gastroenterol 2014;20:9675-9690.

6. Sandborn WJ. Current directions in IBD therapy: what goals are feasible with biological modifiers? Gastroenterology 2008; 135:1442-1447.

7. Lichtenstein GR, Yan S, Bala M, Blank M, Sands BE. Infliximab maintenance treatment reduces hospitalizations, surgeries, and procedures in fistulizing Crohn's disease. Gastroenterology 2005;128:862-869.

8. Colombel JF, Rutgeerts PJ, Sandborn WJ, et al. Adalimumab induces deep remission in patients with Crohn's disease. Clin Gastroenterol Hepatol 2014;12:414-422.

9. D'haens G, Van Deventer S, Van Hogezand R, et al. Endoscopic and histological healing with infliximab anti-tumor necrosis factor antibodies in Crohn's disease: a European multicenter trial. Gastroenterology 1999;116:1029-1034.

10. Rutgeerts P, Van Assche G, Sandborn WJ, et al. Adalimumab induces and maintains mucosal healing in patients with Crohn's disease: data from the EXTEND trial. Gastroenterology 2012; 142:1102-1111.

11. Hanauer SB, Feagan BG, Lichtenstein GR, et al. Maintenance infliximab for Crohn's disease: the ACCENT I randomised 
trial. Lancet 2002;359:1541-1549.

12. Colombel JF, Sandborn WJ, Rutgeerts P, et al. Adalimumab for maintenance of clinical response and remission in patients with Crohn's disease: the CHARM trial. Gastroenterology 2007;132:52-65.

13. Schreiber S. Certolizumab pegol for the treatment of Crohn's disease. Therap Adv Gastroenterol 2011;4:375-389.

14. Schreiber S, Khaliq-Kareemi M, Lawrance IC, et al. Maintenance therapy with certolizumab pegol for Crohn's disease. N Engl J Med 2007;357:239-250.

15. Orlando A, Armuzzi A, Papi C, et al. The Italian Society of Gastroenterology (SIGE) and the Italian Group for the study of inflammatory bowel disease (IG-IBD) clinical practice guidelines: the use of tumor necrosis factor-alpha antagonist therapy in inflammatory bowel disease. Dig Liver Dis 2011;43:1-20.

16. Sandborn WJ, Feagan BG, Rutgeerts P, et al. Vedolizumab as induction and maintenance therapy for Crohn's disease. $\mathrm{N}$ Engl J Med 2013;369:711-721.

17. Sands BE, Feagan BG, Rutgeerts P, et al. Effects of vedolizumab induction therapy for patients with Crohn's disease in whom tumor necrosis factor antagonist treatment failed. Gastroenterology 2014;147:618-627.

18. Gomollón F, Dignass A, Annese V, et al. 3rd European evidence-based consensus on the diagnosis and management of Crohn's disease 2016. Part 1: diagnosis and medical management. J Crohns Colitis 2017;11:3-25.

19. Sandborn WJ, Gasink C, Gao LL, et al. Ustekinumab induction and maintenance therapy in refractory Crohn's disease. N Engl J Med 2012;367:1519-1528.

20. Chen QQ, Yan L, Wan J. Select a suitable treatment strategy for Crohn's disease: step-up or top-down. EXCLI J 2014;13: 111-122.

21. D'Haens GR. Top-down therapy for IBD: rationale and requisite evidence. Nat Rev Gastroenterol Hepatol 2010;7:86-92.

22. Navarra SV, Tang B, Lu L, et al. Risk of tuberculosis with antitumor necrosis factor-alpha therapy: substantially higher number of patients at risk in Asia. Int J Rheum Dis 2014;17:291-298.

23. Wei SC. Differences in the public medical insurance systems for inflammatory bowel disease treatment in Asian countries. Intest Res 2016;14:218-223.

24. Lin MV, Blonski W, Lichtenstein GR. What is the optimal therapy for Crohn's disease: step-up or top-down? Expert Rev Gastroenterol Hepatol 2010;4:167-180.

25. Shergill AK, Terdiman JP. Controversies in the treatment of Crohn's disease: the case for an accelerated step-up treatment approach. World J Gastroenterol 2008;14:2670-2677.
26. Beaugerie L, Seksik P, Nion-Larmurier I, Gendre JP, Cosnes J. Predictors of Crohn's disease. Gastroenterology 2006;130: 650-656.

27. Sands BE, Arsenault JE, Rosen MJ, et al. Risk of early surgery for Crohn's disease: implications for early treatment strategies. Am J Gastroenterol 2003;98:2712-2718.

28. Miheller P, Kiss LS, Juhasz M, Mandel M, Lakatos PL. Recommendations for identifying Crohn's disease patients with poor prognosis. Expert Rev Clin Immunol 2013;9:65-75.

29. Aniwan S, Park SH, Loftus EV Jr. Epidemiology, natural history, and risk stratification of Crohn's disease. Gastroenterol Clin North Am 2017;46:463-480.

30. Mahid SS, Minor KS, Stevens PL, Galandiuk S. The role of smoking in Crohn's disease as defined by clinical variables. Dig Dis Sci 2007;52:2897-2903.

31. Sandborn WJ. Crohn's disease evaluation and treatment: clinical decision tool. Gastroenterology 2014;147:702-705.

32. Oh EH, Oh K, Han M, et al. Early anti-TNF/immunomodulator therapy is associated with better long-term clinical outcomes in Asian patients with Crohn's disease with poor prognostic factors. PLoS One 2017;12:e0177479.

33. D'Haens G, Baert F, van Assche G, et al. Early combined immunosuppression or conventional management in patients with newly diagnosed Crohn's disease: an open randomized trial. Lancet 2008;371:660-667.

34. Colombel JF, Sandborn WJ, Reinisch W, et al. Infliximab, azathioprine, or combination therapy for Crohn's disease. N Engl J Med 2010;362:1383-1395.

35. Panaccione R, Ghosh S, Middleton S, et al. Combination therapy with infliximab and azathioprine is superior to monotherapy with either agent in ulcerative colitis. Gastroenterology 2014;146:392-400.

36. Jones JL, Kaplan GG, Peyrin-Biroulet L, et al. Effects of concomitant immunomodulator therapy on efficacy and safety of anti-tumor necrosis factor therapy for Crohn's disease: a meta-analysis of placebo-controlled trials. Clin Gastroenterol Hepatol 2015;13:2233-2240.

37. Colombel JF, Jharap B, Sandborn WJ, et al. Effects of concomitant immunomodulators on the pharmacokinetics, efficacy and safety of adalimumab in patients with Crohn's disease or ulcerative colitis who had failed conventional therapy. Aliment Pharmacol Ther 2017;45:50-62.

38. Ben-Horin S, Waterman M, Kopylov U, et al. Addition of an immunomodulator to infliximab therapy eliminates antidrug antibodies in serum and restores clinical response of patients with inflammatory bowel disease. Clin Gastroenterol Hepa- 
tol 2013;11:444-447.

39. Strik AS, van den Brink GR, Ponsioen C, Mathot R, Löwenberg M, D'Haens GR. Suppression of anti-drug antibodies to infliximab or adalimumab with the addition of an immunomodulator in patients with inflammatory bowel disease. Aliment Pharmacol Ther 2017;45:1128-1134.

40. Feagan BG, McDonald JW, Panaccione R, et al. Methotrexate in combination with infliximab is no more effective than infliximab alone in patients with Crohn's disease. Gastroenterology 2014;146:681-688.

41. Van Assche G, Magdelaine-Beuzelin C, D'Haens G, et al. Withdrawal of immunosuppression in Crohn's disease treated with scheduled infliximab maintenance: a randomized trial. Gastroenterology 2008;134:1861-1868.

42. Roblin X, Boschetti G, Williet N, et al. Azathioprine dose reduction in inflammatory bowel disease patients on combination therapy: an open-label, prospective and randomised clinical trial. Aliment Pharmacol Ther 2017;46:142-149.

43. Bots S, Gecse K, Barclay M, D’Haens G. Combination immunosuppression in IBD. Inflamm Bowel Dis 2018;24:539-545.

44. Hanauer SB, Wagner CL, Bala M, et al. Incidence and importance of antibody responses to infliximab after maintenance or episodic treatment in Crohn's disease. Clin Gastroenterol Hepatol 2004;2:542-553.

45. Sands BE, Anderson FH, Bernstein CN, et al. Infliximab maintenance therapy for fistulizing Crohn's disease. N Engl J Med 2004;350:876-885.

46. Torres J, Boyapati RK, Kennedy NA, Louis E, Colombel JF, Satsangi J. Systematic review of effects of withdrawal of immunomodulators or biologic agents from patients with inflammatory bowel disease. Gastroenterology 2015;149:17161730.

47. Louis E, Mary JY, Vernier-Massouille G, et al. Maintenance of remission among patients with Crohn's disease on antimetabolite therapy after infliximab therapy is stopped. Gastroenterology 2012;142:63-70.

48. Frias Gomes C, Colombel JF, Torres J. De-escalation of therapy in inflammatory bowel disease. Curr Gastroenterol Rep 2018;20:35.

49. Sorrentino D, Paviotti A, Terrosu G, Avellini C, Geraci M, Zarifi D. Low-dose maintenance therapy with infliximab prevents postsurgical recurrence of Crohn's disease. Clin Gastroenterol Hepatol 2010;8:591-599.

50. Van Steenbergen S, Bian S, Vermeire S, Van Assche G, Gils A, Ferrante M. Dose de-escalation to adalimumab $40 \mathrm{mg}$ every 3 weeks in patients with Crohn's disease: a nested case-con- trol study. Aliment Pharmacol Ther 2017;45:923-932.

51. Kawalec P, Mikrut A, Wiśniewska N, Pilc A. Tumor necrosis factor-alpha antibodies (infliximab, adalimumab and certolizumab) in Crohn's disease: systematic review and meta-analysis. Arch Med Sci 2013;9:765-779.

52. Feagan BG, Schwartz D, Danese S, et al. Efficacy of vedolizumab in fistulising Crohn's disease: exploratory analyses of data from GEMINI 2. J Crohns Colitis 2018;12:621-626.

53. Ji CC, Takano S. Clinical efficacy of adalimumab versus infliximab and the factors associated with recurrence or aggravation during treatment of anal fistulas in Crohn's disease. Intest Res 2017;15:182-186.

54. Panés J, García-Olmo D, Van Assche G, et al. Expanded allogeneic adipose-derived mesenchymal stem cells (Cx601) for complex perianal fistulas in Crohn's disease: a phase 3 randomised, double-blind controlled trial. Lancet 2016;388:12811290.

55. Rutgeerts P, Sandborn WJ, Feagan BG, et al. Infliximab for induction and maintenance therapy for ulcerative colitis. $N$ Engl J Med 2005;353:2462-2476.

56. Sandborn WJ, van Assche G, Reinisch W, et al. Adalimumab induces and maintains clinical remission in patients with moderate-to-severe ulcerative colitis. Gastroenterology 2012 142:257-265.

57. Sandborn WJ, Feagan BG, Marano C, et al. Subcutaneous golimumab induces clinical response and remission in patients with moderate-to-severe ulcerative colitis. Gastroenterology 2014;146:85-95.

58. Sandborn WJ, Feagan BG, Marano C, et al. Subcutaneous golimumab maintains clinical response in patients with moderate-to-severe ulcerative colitis. Gastroenterology 2014;146: 96-109.

59. Feagan BG, Rutgeerts P, Sands BE, et al. Vedolizumab as induction and maintenance therapy for ulcerative colitis. N Engl J Med 2013;369:699-710.

60. Järnerot G, Hertervig E, Friis-Liby I, et al. Infliximab as rescue therapy in severe to moderately severe ulcerative colitis: a randomized, placebo-controlled study. Gastroenterology 2005; 128:1805-1811.

61. García-López S, Gomollón-García F, Pérez-Gisbert J. Cyclosporine in the treatment of severe attack of ulcerative colitis a systematic review. Gastroenterol Hepatol 2005;28:607-614.

62. Laharie D, Bourreille A, Branche J, et al. Ciclosporin versus infliximab in patients with severe ulcerative colitis refractory to intravenous steroids: a parallel, open-label randomised controlled trial. Lancet 2012;380:1909-1915. 
63. Williams JG, Alam MF, Alrubaiy L, et al. Infliximab versus ciclosporin for steroid-resistant acute severe ulcerative colitis (CONSTRUCT): a mixed methods, open-label, pragmatic randomised trial. Lancet Gastroenterol Hepatol 2016;1:15-24.

64. Chen JH, Andrews JM, Kariyawasam V, et al. Review article: acute severe ulcerative colitis - evidence-based consensus statements. Aliment Pharmacol Ther 2016;44:127-144.

65. Peyrin-Biroulet L, Van Assche G, Gómez-Ulloa D, et al. Systematic review of tumor necrosis factor antagonists in extraintestinal manifestations in inflammatory bowel disease. Clin Gastroenterol Hepatol 2017;15:25-36.

66. Tadbiri S, Peyrin-Biroulet L, Serrero M, et al. Impact of vedolizumab therapy on extra-intestinal manifestations in patients with inflammatory bowel disease: a multicentre cohort study nested in the OBSERV-IBD cohort. Aliment Pharmacol Ther 2018;47:485-493.

67. Colombel JF, Sands BE, Rutgeerts P, et al. The safety of vedolizumab for ulcerative colitis and Crohn's disease. Gut 2017; 66:839-851.

68. Amiot A, Serrero M, Peyrin-Biroulet L, et al. One-year effectiveness and safety of vedolizumab therapy for inflammatory bowel disease: a prospective multicenter cohort study. Aliment Pharmacol Ther 2017;46:310-321.

69. Sands BE, Sandborn WJ, Van Assche G, et al. Vedolizumab as induction and maintenance therapy for Crohn's disease in patients naïve to or who have failed tumor necrosis factor antagonist therapy. Inflamm Bowel Dis 2017;23:97-106.

70. Gottlieb AB, Kalb RE, Langley RG, et al. Safety observations in 12095 patients with psoriasis enrolled in an international registry (PSOLAR): experience with infliximab and other systemic and biologic therapies. J Drugs Dermatol 2014;13:14411448.

71. Tsai TF, Ho V, Song M, et al. The safety of ustekinumab treatment in patients with moderate-to-severe psoriasis and latent tuberculosis infection. Br J Dermatol 2012;167:1145-1152.

72. Papp K, Gottlieb AB, Naldi L, et al. Safety surveillance for ustekinumab and other psoriasis treatments from the Psoriasis Longitudinal Assessment and Registry (PSOLAR). J Drugs Dermatol 2015;14:706-714.

73. McInnes IB, Kavanaugh A, Gottlieb AB, et al. Efficacy and safety of ustekinumab in patients with active psoriatic arthritis: 1 year results of the phase 3, multicentre, double-blind, placebo-controlled PSUMMIT 1 trial. Lancet 2013;382:780789.

74. Ritchlin C, Rahman P, Kavanaugh A, et al. Efficacy and safety of the anti-IL-12/23 p40 monoclonal antibody, ustekinumab, in patients with active psoriatic arthritis despite conventional non-biological and biological anti-tumour necrosis factor therapy: 6-month and 1-year results of the phase 3, multicentre, double-blind, placebo-controlled, randomised PSUMMIT 2 trial. Ann Rheum Dis 2014;73:990-999.

75. Lynch M, Roche L, Horgan M, Ahmad K, Hackett C, Ramsay B. Peritoneal tuberculosis in the setting of ustekinumab treatment for psoriasis. JAAD Case Rep 2017;3:230-232.

76. Sánchez-Moya AI, Daudén E. Peripheral lymph node recurrence of tuberculosis after ustekinumab treatment. Arch Dermatol 2012;148:1332-1333.

77. Scott FI, Lichtenstein GR. Biosimilars in the treatment of inflammatory bowel disease: supporting evidence in 2017. Curr Treat Options Gastroenterol 2018;16:147-164.

78. Avila-Ribeiro P, Fiorino G, Danese S. The experience with biosimilars of infliximab in inflammatory bowel disease. Curr Pharm Des 2017;23:6759-6769.

79. Cohen HP, Blauvelt A, Rifkin RM, Danese S, Gokhale SB, Woollett G. Switching reference medicines to biosimilars: a systematic literature review of clinical outcomes. Drugs 2018;78: 463-478.

80. Fiorino G, Manetti N, Armuzzi A, et al. The PROSIT-BIO cohort: a prospective observational study of patients with inflammatory bowel disease treated with infliximab biosimilar. Inflamm Bowel Dis 2017;23:233-243.

81. Schmitz EMH, Boekema PJ, Straathof JWA, et al. Switching from infliximab innovator to biosimilar in patients with inflammatory bowel disease: a 12-month multicentre observational prospective cohort study. Aliment Pharmacol Ther 2018; 47:356-363.

82. Park SH, Kim YH, Lee JH, et al. Post-marketing study of biosimilar infliximab (CT-P13) to evaluate its safety and efficacy in Korea. Expert Rev Gastroenterol Hepatol 2015;9 Suppl 1: 35-44.

83. Kurti Z, Gonczi L, Lakatos PL. Progress with infliximab biosimilars for inflammatory bowel disease. Expert Opin Biol Ther 2018;18:633-640.

84. Peyrin-Biroulet L, Sandborn W, Sands BE, et al. Selecting therapeutic targets in inflammatory bowel disease (STRIDE): determining therapeutic goals for treat-to-target. Am J Gastroenterol 2015;110:1324-1338.

85. Levesque BG, Sandborn WJ, Ruel J, Feagan BG, Sands BE, Colombel JF. Converging goals of treatment of inflammatory bowel disease from clinical trials and practice. Gastroenterology 2015;148:37-51.

86. Walsh AJ, Bryant RV, Travis SP. Current best practice for dis- 
ease activity assessment in IBD. Nat Rev Gastroenterol Hepatol 2016;13:567-579.

87. Best WR, Becktel JM, Singleton JW, Kern F Jr. Development of a Crohn's disease activity index: national cooperative Crohn's disease study. Gastroenterology 1976;70:439-444.

88. Thia KT, Sandborn WJ, Lewis JD, et al. Defining the optimal response criteria for the Crohn's disease activity index for induction studies in patients with mildly to moderately active Crohn's disease. Am J Gastroenterol 2008;103:3123-3131.

89. Harvey RF, Bradshaw JM. A simple index of Crohn's-disease activity. Lancet 1980;1:514.

90. Sandborn WJ, Sands BE, Wolf DC, et al. Repifermin (keratinocyte growth factor-2) for the treatment of active ulcerative colitis: a randomized, double-blind, placebo-controlled, doseescalation trial. Aliment Pharmacol Ther 2003;17:1355-1364.

91. Schroeder KW, Tremaine WJ, Ilstrup DM. Coated oral 5-aminosalicylic acid therapy for mildly to moderately active ulcerative colitis: a randomized study. N Engl J Med 1987;317:16251629.

92. Frøslie KF, Jahnsen J, Moum BA, Vatn MH; IBSEN Group. Mucosal healing in inflammatory bowel disease: results from a Norwegian population-based cohort. Gastroenterology 2007; 133:412-422

93. Rutgeerts P, Diamond RH, Bala M, et al. Scheduled maintenance treatment with infliximab is superior to episodic treatment for the healing of mucosal ulceration associated with Crohn's disease. Gastrointest Endosc 2006;63:433-442.

94. Schnitzler F, Fidder H, Ferrante M, et al. Mucosal healing predicts long-term outcome of maintenance therapy with infliximab in Crohn's disease. Inflamm Bowel Dis 2009;15:12951301.

95. Colombel JF, Rutgeerts P, Reinisch W, et al. Early mucosal healing with infliximab is associated with improved long-term clinical outcomes in ulcerative colitis. Gastroenterology 2011; 141:1194-1201.

96. Jones J, Loftus EV Jr, Panaccione R, et al. Relationships between disease activity and serum and fecal biomarkers in patients with Crohn's disease. Clin Gastroenterol Hepatol 2008;6:12181224.

97. Peyrin-Biroulet L, Reinisch W, Colombel JF, et al. Clinical disease activity, C-reactive protein normalisation and mucosal healing in Crohn's disease in the SONIC trial. Gut 2014;63:8895.

98. Boschetti G, Garnero P, Moussata D, et al. Accuracies of serum and fecal S100 proteins (calprotectin and calgranulin C) to predict the response to TNF antagonists in patients with
Crohn's disease. Inflamm Bowel Dis 2015;21:331-336.

99. Cornillie F, Hanauer SB, Diamond RH, et al. Postinduction serum infliximab trough level and decrease of C-reactive protein level are associated with durable sustained response to infliximab: a retrospective analysis of the ACCENT I trial. Gut 2014;63:1721-1727.

100. Molander P, af Björkesten CG, Mustonen H, et al. Fecal calprotectin concentration predicts outcome in inflammatory bowel disease after induction therapy with TNFalpha blocking agents. Inflamm Bowel Dis 2012;18:2011-2017.

101. Kiss LS, Szamosi T, Molnar T, et al. Early clinical remission and normalisation of CRP are the strongest predictors of efficacy, mucosal healing and dose escalation during the first year of adalimumab therapy in Crohn's disease. Aliment Pharmacol Ther 2011;34:911-922.

102. Guidi L, Marzo M, Andrisani G, et al. Faecal calprotectin assay after induction with anti-tumour necrosis factor alpha agents in inflammatory bowel disease: prediction of clinical response and mucosal healing at one year. Dig Liver Dis 2014; 46:974-979.

103. Iwasa R, Yamada A, Sono K, Furukawa R, Takeuchi K, Suzuki Y. C-reactive protein level at 2 weeks following initiation of infliximab induction therapy predicts outcomes in patients with ulcerative colitis: a 3 year follow-up study. BMC Gastroenterol 2015;15:103.

104. Panes J, Bouhnik Y, Reinisch W, et al. Imaging techniques for assessment of inflammatory bowel disease: joint ECCO and ESGAR evidence-based consensus guidelines. J Crohns Colitis 2013;7:556-585.

105. Efthymiou A, Viazis N, Mantzaris G, et al. Does clinical response correlate with mucosal healing in patients with Crohn's disease of the small bowel? A prospective, case-series study using wireless capsule endoscopy. Inflamm Bowel Dis 2008; 14:1542-1547.

106. Hall B, Holleran G, Chin JL, et al. A prospective 52 week mucosal healing assessment of small bowel Crohn's disease as detected by capsule endoscopy. J Crohns Colitis 2014;8:16011609.

107. Hall BJ, Holleran GE, Smith SM, Mahmud N, McNamara DA. A prospective 12-week mucosal healing assessment of small bowel Crohn's disease as detected by capsule endoscopy. Eur J Gastroenterol Hepatol 2014;26:1253-1259.

108. Kopylov U, Yablecovitch D, Lahat A, et al. Detection of small bowel mucosal healing and deep remission in patients with known small bowel Crohn's disease using biomarkers, capsule endoscopy, and imaging. Am J Gastroenterol 2015;110: 


\section{$1316-1323$}

109. Yang L, Ge ZZ, Gao YJ, et al. Assessment of capsule endoscopy scoring index, clinical disease activity, and C-reactive protein in small bowel Crohn's disease. J Gastroenterol Hepatol 2013;28:829-833.

110. Carvalho PB, Rosa B, Cotter J. Mucosal healing in Crohn's disease - are we reaching as far as possible with capsule endoscopy? J Crohns Colitis 2014;8:1566-1567.

111. Niv Y. Small-bowel mucosal healing assessment by capsule endoscopy as a predictor of long-term clinical remission in patients with Crohn's disease: a systematic review and metaanalysis. Eur J Gastroenterol Hepatol 2017;29:844-848.

112. Kopylov U, Yung DE, Engel T, et al. Diagnostic yield of capsule endoscopy versus magnetic resonance enterography and small bowel contrast ultrasound in the evaluation of small bowel Crohn's disease: systematic review and meta-analysis. Dig Liver Dis 2017;49:854-863.

113. Cotter J, Dias de Castro F, Magalhães J, Moreira MJ, Rosa B. Validation of the Lewis score for the evaluation of small-bowel Crohn's disease activity. Endoscopy 2015;47:330-335.

114. Niv Y, Ilani S, Levi Z, et al. Validation of the capsule endoscopy Crohn's disease activity index (CECDAI or Niv score): a multicenter prospective study. Endoscopy 2012;44:21-26.

115. Enns RA, Hookey L, Armstrong D, et al. Clinical practice guidelines for the use of video capsule endoscopy. Gastroenterology 2017;152:497-514.

116. Cheifetz AS, Kornbluth AA, Legnani P, et al. The risk of retention of the capsule endoscope in patients with known or suspected Crohn's disease. Am J Gastroenterol 2006;101:22182222.

117. Liao Z, Gao R, Xu C, Li ZS. Indications and detection, completion, and retention rates of small-bowel capsule endoscopy: a systematic review. Gastrointest Endosc 2010;71:280-286.

118. Postgate AJ, Burling D, Gupta A, Fitzpatrick A, Fraser C. Safety, reliability and limitations of the given patency capsule in patients at risk of capsule retention: a 3-year technical review. Dig Dis Sci 2008;53:2732-2738.

119. Höög CM, Bark LÅ, Arkani J, Gorsetman J, Broström O, Sjöqvist U. Capsule retentions and incomplete capsule endoscopy examinations: an analysis of 2300 examinations. Gastroenterol Res Pract 2012;2012:518718.

120. Ye CA, Gao YJ, Ge ZZ, et al. PillCam colon capsule endoscopy versus conventional colonoscopy for the detection of severity and extent of ulcerative colitis. J Dig Dis 2013;14:117124.

121. Hosoe N, Matsuoka K, Naganuma M, et al. Applicability of second-generation colon capsule endoscope to ulcerative colitis: a clinical feasibility study. J Gastroenterol Hepatol 2013; 28:1174-1179.

122. Meister T, Heinzow HS, Domagk D, et al. Colon capsule endoscopy versus standard colonoscopy in assessing disease activity of ulcerative colitis: a prospective trial. Tech Coloproctol 2013;17:641-646.

123. Sung J, Ho KY, Chiu HM, Ching J, Travis S, Peled R. The use of Pillcam Colon in assessing mucosal inflammation in ulcerative colitis: a multicenter study. Endoscopy 2012;44:754-758.

124. Collins PD. Video capsule endoscopy in inflammatory bowel disease. World J Gastrointest Endosc 2016;8:477-488.

125. Reenaers C, Mary JY, Nachury M, et al. Outcomes 7 years after infliximab withdrawal for patients with Crohn's disease in sustained remission. Clin Gastroenterol Hepatol 2018;16:234243.

126. Doherty G, Katsanos KH, Burisch J, et al. European Crohn's and Colitis Organisation Topical Review on Treatment Withdrawal ['Exit Strategies'] in inflammatory bowel disease. J Crohns Colitis 2018;12:17-31.

127. Treton X, Bouhnik Y, Mary JY, et al. Azathioprine withdrawal in patients with Crohn's disease maintained on prolonged remission: a high risk of relapse. Clin Gastroenterol Hepatol 2009;7:80-85.

128. O'Donoghue DP, Dawson AM, Powell-Tuck J, Bown RL, Lennard-Jones JE. Double-blind withdrawal trial of azathioprine as maintenance treatment for Crohn's disease. Lancet 1978; 2:955-957.

129. Hawthorne AB, Logan RF, Hawkey CJ, et al. Randomised controlled trial of azathioprine withdrawal in ulcerative colitis. BMJ 1992;305:20-22.

130. Lémann M, Mary JY, Colombel JF, et al. A randomized, double-blind, controlled withdrawal trial in Crohn's disease patients in long-term remission on azathioprine. Gastroenterology 2005;128:1812-1818.

131. Clarke K, Regueiro M. Stopping immunomodulators and biologics in inflammatory bowel disease patients in remission. Inflamm Bowel Dis 2012;18:174-179.

132. Pittet V, Froehlich F, Maillard MH, et al. When do we dare to stop biological or immunomodulatory therapy for Crohn's disease? Results of a multidisciplinary European expert panel. J Crohns Colitis 2013;7:820-826.

133. Kirchgesner J, Beaugerie L, Carrat F, et al. Impact on life expectancy of withdrawing thiopurines in patients with Crohn's disease in sustained clinical remission: a lifetime risk-benefit analysis. PLoS One 2016;11:e0157191. 
134. Chouchana L, Roche D, Narjoz C, et al. Screening of TPMT deficiency by phenotyping and genotyping: a retrospective study among 1,500 IBD patients in France. Gastroenterology 2011;140(5 Suppl 1):S281-S282.

135. Chevaux JB, Peyrin-Biroulet L, Sparrow MP. Optimizing thiopurine therapy in inflammatory bowel disease. Inflamm Bowel Dis 2011;17:1428-1435.

136. Kham SK, Soh CK, Liu TC, et al. Thiopurine S-methyltransferase activity in three major Asian populations: a population-based study in Singapore. Eur J Clin Pharmacol 2008;64:373-379.

137. Yang SK, Hong M, Baek J, et al. A common missense variant in NUDT15 confers susceptibility to thiopurine-induced leukopenia. Nat Genet 2014;46:1017-1020.

138. Takatsu N, Matsui T, Murakami Y, et al. Adverse reactions to azathioprine cannot be predicted by thiopurine S-methyltransferase genotype in Japanese patients with inflammatory bowel disease. J Gastroenterol Hepatol 2009;24:1258-1264.

139. Fangbin Z, Xiang G, Minhu C, et al. Should thiopurine methyltransferase genotypes and phenotypes be measured before thiopurine therapy in patients with inflammatory bowel disease? Ther Drug Monit 2012;34:695-701.

140. Moriyama T, Nishii R, Perez-Andreu V, et al. NUDT15 polymorphisms alter thiopurine metabolism and hematopoietic toxicity. Nat Genet 2016;48:367-373.

141. Yang JJ, Landier W, Yang W, et al. Inherited NUDT15 variant is a genetic determinant of mercaptopurine intolerance in children with acute lymphoblastic leukemia. J Clin Oncol 2015;33:1235-1242.

142. Zhang AL, Yang J, Wang H, Lu JL, Tang S, Zhang XJ. Association of NUDT15 c.415C $>$ T allele and thiopurine-induced leukocytopenia in Asians: a systematic review and meta-analysis. Ir J Med Sci 2018;187:145-153.

143. Steenholdt C, Bendtzen K, Brynskov J, Thomsen OØ, Ainsworth MA. Cut-off levels and diagnostic accuracy of infliximab trough levels and anti-infliximab antibodies in Crohn's disease. Scand J Gastroenterol 2011;46:310-318.

144. Bortlik M, Duricova D, Malickova K, et al. Infliximab trough levels may predict sustained response to infliximab in patients with Crohn's disease. J Crohns Colitis 2013;7:736-743.

145. Adedokun OJ, Sandborn WJ, Feagan BG, et al. Association between serum concentration of infliximab and efficacy in adult patients with ulcerative colitis. Gastroenterology 2014; 147:1296-1307.

146. Levesque BG, Greenberg GR, Zou G, et al. A prospective cohort study to determine the relationship between serum infliximab concentration and efficacy in patients with luminal
Crohn's disease. Aliment Pharmacol Ther 2014;39:1126-1135.

147. Vande Casteele N, Khanna R, Levesque BG, et al. The relationship between infliximab concentrations, antibodies to infliximab and disease activity in Crohn's disease. Gut 2015; 64:1539-1545.

148. Reinisch W, Colombel JF, Sandborn WJ, et al. Factors associated with short- and long-term outcomes of therapy for Crohn's disease. Clin Gastroenterol Hepatol 2015;13:539-547.

149. Vande Casteele N, Ferrante M, Van Assche G, et al. Trough concentrations of infliximab guide dosing for patients with inflammatory bowel disease. Gastroenterology 2015;148:13201329.

150. Karmiris K, Paintaud G, Noman M, et al. Influence of trough serum levels and immunogenicity on long-term outcome of adalimumab therapy in Crohn's disease. Gastroenterology 2009;137:1628-1640

151. Roblin X, Marotte H, Rinaudo M, et al. Association between pharmacokinetics of adalimumab and mucosal healing in patients with inflammatory bowel diseases. Clin Gastroenterol Hepatol 2014;12:80-84.

152. Mazor Y, Almog R, Kopylov U, et al. Adalimumab drug and antibody levels as predictors of clinical and laboratory response in patients with Crohn's disease. Aliment Pharmacol Ther 2014;40:620-628.

153. Roblin X, Rinaudo M, Del Tedesco E, et al. Development of an algorithm incorporating pharmacokinetics of adalimumab in inflammatory bowel diseases. Am J Gastroenterol 2014; 109:1250-1256.

154. Morita Y, Imaeda H, Nishida A, et al. Association between serum adalimumab concentrations and endoscopic disease activity in patients with Crohn's disease. J Gastroenterol Hepatol 2016;31:1831-1836.

155. Ungar B, Levy I, Yavne Y, et al. Optimizing anti-TNF-alpha therapy: serum levels of infliximab and adalimumab are associated with mucosal healing in patients with inflammatory bowel diseases. Clin Gastroenterol Hepatol 2016;14:550-557.

156. Yanai H, Lichtenstein L, Assa A, et al. Levels of drug and antidrug antibodies are associated with outcome of interventions after loss of response to infliximab or adalimumab. Clin Gastroenterol Hepatol 2015;13:522-530.

157. Vande Casteele N, Gils A, Singh S, et al. Antibody response to infliximab and its impact on pharmacokinetics can be transient. Am J Gastroenterol 2013;108:962-971.

158. Dignass A, Van Assche G, Lindsay JO, et al. The second European evidence-based consensus on the diagnosis and management of Crohn's disease: current management. J Crohns 
Colitis 2010;4:28-62.

159. Candy S, Wright J, Gerber M, Adams G, Gerig M, Goodman R. A controlled double blind study of azathioprine in the management of Crohn's disease. Gut 1995;37:674-678.

160. Present DH, Korelitz BI, Wisch N, Glass JL, Sachar DB, Pasternack BS. Treatment of Crohn's disease with 6-mercaptopurine: a long-term, randomized, double-blind study. N Engl J Med 1980;302:981-987.

161. Dubinsky MC, Lamothe S, Yang HY, et al. Pharmacogenomics and metabolite measurement for 6-mercaptopurine therapy in inflammatory bowel disease. Gastroenterology 2000; 118:705-713.

162. Osterman MT, Kundu R, Lichtenstein GR, Lewis JD. Association of 6-thioguanine nucleotide levels and inflammatory bowel disease activity: a meta-analysis. Gastroenterology 2006;130:1047-1053.

163. Hindorf U, Lindqvist M, Hildebrand H, Fagerberg U, Almer S. Adverse events leading to modification of therapy in a large cohort of patients with inflammatory bowel disease. Aliment Pharmacol Ther 2006;24:331-342.

164. Gearry RB, Barclay ML. Azathioprine and 6-mercaptopurine pharmacogenetics and metabolite monitoring in inflammatory bowel disease. J Gastroenterol Hepatol 2005;20:11491157.

165. Haines ML, Ajlouni Y, Irving PM, et al. Clinical usefulness of therapeutic drug monitoring of thiopurines in patients with inadequately controlled inflammatory bowel disease. Inflamm Bowel Dis 2011;17:1301-1307.

166. Kennedy NA, Asser TL, Mountifield RE, Doogue MP, Andrews JM, Bampton PA. Thiopurine metabolite measurement leads to changes in management of inflammatory bowel disease. Intern Med J 2013;43:278-286.

167. Baert F, Noman M, Vermeire S, et al. Influence of immunogenicity on the long-term efficacy of infliximab in Crohn's disease. N Engl J Med 2003;348:601-608.

168. Yarur AJ, Kubiliun MJ, Czul F, et al. Concentrations of 6-thioguanine nucleotide correlate with trough levels of infliximab in patients with inflammatory bowel disease on combination therapy. Clin Gastroenterol Hepatol 2015;13:1118-1124.

169. Ott JJ, Stevens GA, Groeger J, Wiersma ST. Global epidemiology of hepatitis B virus infection: new estimates of age-specific HBsAg seroprevalence and endemicity. Vaccine 2012; 30:2212-2219.

170. Cheon JH. Understanding the complications of anti-tumor necrosis factor therapy in East Asian patients with inflammatory bowel disease. J Gastroenterol Hepatol 2017;32:769-777.
171. Rahier JF, Magro F, Abreu C, et al. Second European evidencebased consensus on the prevention, diagnosis and management of opportunistic infections in inflammatory bowel disease. J Crohns Colitis 2014;8:443-468.

172. Loras C, Gisbert JP, Mínguez M, et al. Liver dysfunction related to hepatitis $\mathrm{B}$ and $\mathrm{C}$ in patients with inflammatory bowel disease treated with immunosuppressive therapy. Gut 2010; 59:1340-1346.

173. Park SH, Yang SK, Lim YS, et al. Clinical courses of chronic hepatitis B virus infection and inflammatory bowel disease in patients with both diseases. Inflamm Bowel Dis 2012;18: 2004-2010.

174. Lin JN, Lai CH, Chen YH, et al. Risk factors for extra-pulmonary tuberculosis compared to pulmonary tuberculosis. Int J Tuberc Lung Dis 2009;13:620-625.

175. Kulchavenya E. Extrapulmonary tuberculosis: are statistical reports accurate? Ther Adv Infect Dis 2014;2:61-70.

176. Lim CH, Chen HH, Chen YH, et al. The risk of tuberculosis disease in rheumatoid arthritis patients on biologics and targeted therapy: a 15-year real world experience in Taiwan. PLoS One 2017;12:e0178035.

177. Weng MT, Wei SC, Lin CC, et al. Seminar report from the 2014 Taiwan Society of Inflammatory Bowel Disease (TSIBD) spring forum (May 24th, 2014): Crohn's disease versus intestinal tuberculosis infection. Intest Res 2015;13:6-10.

178. Horsburgh CR Jr, Rubin EJ. Clinical practice: latent tuberculosis infection in the United States. N Engl J Med 201 1;364:1441 1448.

179. Lee J, Kim E, Jang EJ, et al. Efficacy of treatment for latent tuberculosis in patients undergoing treatment with a tumor necrosis factor antagonist. Ann Am Thorac Soc 2017;14:690-697.

180. Park DI, Hisamatsu T, Chen M, et al. Asian Organization for Crohn's and Colitis and Asia Pacific Association of Gastroenterology consensus on tuberculosis infection in patients with inflammatory bowel disease receiving anti-tumor necrosis factor treatment. Part 2: management. J Gastroenterol Hepatol 2018;33:30-36.

181. Naderi HR, Sheybani F, Rezaei Pajand S. How should we manage latent tuberculosis infection in patients receiving antiTNF-alpha drugs: literature review. Iran Red Crescent Med J 2016;18:e27756.

182. Demir S, Sadi Aykan F, Öztuna D. Latent tuberculosis treatment results in patients that taken TNF-alpha blockers at Ankara Numune Training and Research Hospital Chest Diseases Clinic for last 8 years (2006-2013). Tuberk Toraks 2014;62: 286-290. 